\title{
Modeling and analyzing observed transverse sand bars in the surf zone
}

\author{
F. Ribas, ${ }^{1,2}$ H. E. de Swart, ${ }^{3}$ D. Calvete, ${ }^{1}$ and A. Falqués ${ }^{1}$ \\ Received 20 July 2011; revised 7 March 2012; accepted 9 March 2012; published 25 April 2012.
}

[1] A morphodynamic model has been applied to explain the characteristics of transverse sandbars observed in the inner surf zone of open beaches. The model describes the feedback between waves, rollers, depth-averaged currents and bed evolution, so that self-organized processes can develop. The modeled bar characteristics, i.e. wavelength (30-70 m), crest orientation (up-current) and the e-folding growth time (about $12 \mathrm{hr}$ ) are in good agreement with those of observed transverse bars at Noordwijk beach, the Netherlands, but modeled migration speeds (tens of meters per day), turn out to be a factor 2 larger than those observed. The wavelength increases with the distance between the shoreline and the peak of the longshore current and the migration speed is correlated with the maximum longshore current. The model also explains why transverse bar formation at Noordwijk occurs for obliquely incident waves of intermediate heights. Realistic positive feedback leading to formation of up-current oriented bars like those observed is only obtained if a term related to the turbulence sediment resuspension created by the rollers is included in the transport formula. In that case, the depth-averaged sediment concentration decreases seaward across the inner surf zone, enhancing the convergence of sediment transport in the offshore directed flow perturbations that occur over the up-current bars. This offshore current deflection is mainly caused by frictional torques, but the roller radiation stresses also play an important role.

Citation: Ribas, F., H. E. de Swart, D. Calvete, and A. Falqués (2012), Modeling and analyzing observed transverse sand bars in the surf zone, J. Geophys. Res., 117, F02013, doi:10.1029/2011JF002158.

\section{Introduction}

[2] Patches of shore-attached transverse sandbars, spaced at fairly uniform alongshore distances, are frequently observed in the surf zone of open seas. Their alongshore wavelength (spacing between successive crests) ranges from tens to a few hundreds of meters, and their crests can show an oblique orientation with respect to the shore-normal. This description covers different types of surf zone bars that show distinctive characteristics and that probably have different origins. For instance, the transverse bars described in classification schemes of open beaches develop when the horns of a pre-existing crescentic bar weld to the beach [Wright and Short, 1984]. Two recent studies have reported observations of another type of transverse bars along the open beaches of Duck, USA [see Konicki and Holman, 2000] and Noordwijk, Netherlands (see Ribas and Kroon [2007] and Figure 1). This second type of bars was named 'transverse finger bars' to emphasize that they were thin and elongated.

\footnotetext{
${ }^{1}$ Departament de Física Aplicada, Universitat Politècnica de Catalunya, Barcelona, Spain.

${ }^{2}$ Formerly at Institute for Marine and Atmospheric Research Utrecht, Utrecht University, Utrecht, Netherlands.

${ }^{3}$ Institute for Marine and Atmospheric Research Utrecht, Utrecht University, Utrecht, Netherlands.

Copyright 2012 by the American Geophysical Union. 0148-0227/12/2011JF002158
}

The focus of the present study are these transverse finger bars, the origin of which is still not understood.

[3] Analysis of video images of Duck and Noordwijk revealed that on these beaches, characterized by one to three shore-parallel subtidal bars, the transverse finger bars were mostly observed in the inner surf zone, attached to the lowtide shoreline (e.g., see Figure 1). Table 1 summarizes the characteristics of the transverse bars observed at these two sites by Konicki and Holman [2000] and Ribas and Kroon [2007]. Bars occurred more often at Noordwijk than at Duck, with a larger percentage of time with bar presence and a larger number of bars per patch. The wavelength detected at Noordwijk was half the size of that at Duck and bar crests deviated from the shore-normal by $20^{\circ}-30^{\circ}$ in both sites. Typical daily averaged migration rates were up to a few tens of meters per day. Ribas and Kroon [2007] established some relationships between the bar characteristics at Noordwijk and the corresponding bathymetric and offshore wave conditions. Bars typically persisted during a few days, coexisting with steady waves of intermediate heights and oblique incidence. The averaged offshore (18 $\mathrm{m}$ depth) wave conditions during bar presence showed a root mean square height of $0.8 \mathrm{~m}$, a peak period of $5.7 \mathrm{~s}$ and an offshore incidence angle of $49^{\circ}$, measured from the shore-normal. Bars showed an 'up-current orientation', i.e., with respect to the longshore current, the seaward ends of their crests were located further upstream than their shore attachments. Their 


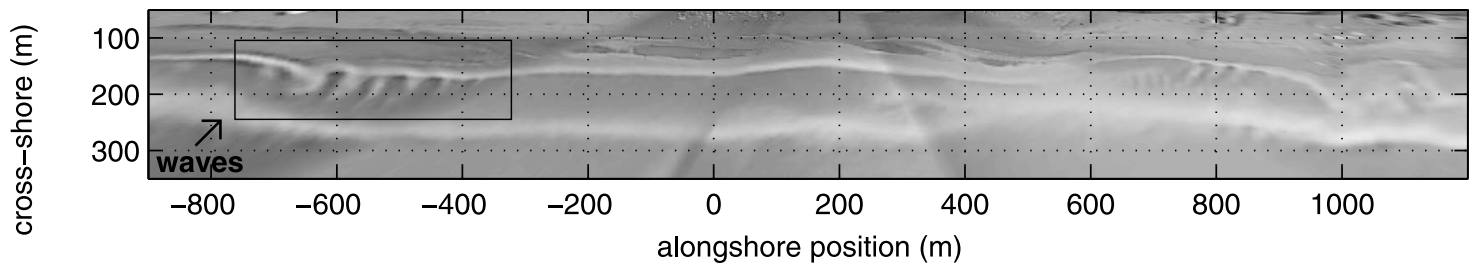

Figure 1. Time-exposure plan view image of Noordwijk beach, the Netherlands, on 22 September 2002 at 11:00 UTC. A patch of surf zone transverse finger bars can be seen at $\mathrm{y}=[-700,-400] \mathrm{m}$ and $x=180 \mathrm{~m}$, attached to the low-tide shoreline.

wavelength showed significant correlation with a proxy for the surf zone width and a highly significant correlation was found between the migration rates and the wave radiation stress component $S_{12}$ (i.e., bar patches migrated in the direction of the longshore current). The underlying bathymetry also influenced Noordwijk bars. For example, crossshore inner trough areas larger than $75 \mathrm{~m}^{2}$ and inner surf zone slopes larger than 0.02 were most favorable to bar growth.

[4] Alongshore rhythmic topography (like the patches of transverse finger bars) are visible prints of physical mechanisms that may dominate the complex surfzone system under certain circumstances and their study allows improved understanding of these governing mechanisms. A possible explanation for transverse finger bar formation is based on the concept of morphodynamic self-organization. Topographic perturbations superimposed on an alongshore uniform beach profile induce hydrodynamic perturbations, which can lead to convergence of sand transport over the bars, hence producing a positive feedback. Linear stability analysis is a convenient tool to investigate the possible feedbacks, yielding information about the shape, growth rate and migration speed of the initially emerging modes. Nonlinear models have then been used to describe the finite amplitude features and to verify the results of the linear stability analysis.

[5] In the early models of transverse finger bar formation based on self-organization [Ribas et al., 2003; Klein and Schuttelaars, 2005; Garnier et al., 2006; van Leeuwen et al., 2006], the modeled shapes and the timescales for growth and migration strongly depended on the specific description of wave propagation and sediment transport. Hence, a quantitative comparison of model results and field observations, which has not been performed so far, is crucial to discriminate between the different potential physical mechanisms proposed for the formation of finger bars. The study of Ribas and Kroon [2007] used the field observations of finger bars at Noordwijk to test qualitatively the results of the existing self-organization models for finger bar formation. Considering that Noordwijk bars were up-current oriented and that they emerged during periods of clearly oblique wave incidence, only one of the existing physical mechanisms remained as a viable explanation for their formation, the so-called 'bed-flow interaction' [Falqués et al., 1996; Ribas et al., 2003]. Table 2 lists examples of results of two studies that included this interaction [Ribas et al., 2003; Garnier et al., 2006]. Modeled wavelengths, growth times and migration rates are consistent with observations reported in Table 1.

[6] The validity of the two models described in Table 2 is rather limited because they are based on strong assumptions, like a uniform beach slope, no full wave refraction and no rollers. Another severe assumption of both models concerns the sediment transport formulation, in particular the crossshore distribution of the depth-integrated volumetric sediment concentration, $\mathcal{C}$. The physical mechanism behind the formation of up-current oriented bars (i.e., the bed-flow interaction) requires that the depth-averaged concentration $\left(\mathcal{C}_{d a}=\mathcal{C} / D\right.$, where $D$ is the water depth) is a decreasing function in the offshore direction [Ribas et al., 2003]. The models in Table 2 assumed an spatially uniform $\mathcal{C}$ so that this condition was obeyed. When more standard sediment transport formulations are employed (e.g., the original Soulsby and van Rijn formula given by Soulsby [1997]), $\mathcal{C}_{d a}$ increases in the seaward direction and the formation of up-current oriented bars is not reproduced.

[7] In order to demonstrate that the bed-flow interaction can indeed explain the formation of the up-current oriented finger bars observed on natural beaches, a physical explanation for the decreasing cross-shore profile of $\mathcal{C}_{d a}$ must be found. An important mechanism that has been so far

Table 1. Characteristics of the Transverse 'Trough Bars' of Duck Beach, USA [Konicki and Holman, 2000] and the Transverse Finger Bars of Noordwijk Beach, the Netherlands [Ribas and Kroon, 2007] ${ }^{\mathrm{a}}$

\begin{tabular}{lccccccccccc}
\hline \multicolumn{1}{c}{ Site } & $d_{50}(\mathrm{~mm})$ & $\Delta z_{s}^{\text {ann }}(\mathrm{m})$ & $H_{\mathrm{rms}}^{\text {ann }}(\mathrm{m})$ & $T_{p}^{\text {ann }}(\mathrm{s})$ & $\left|\theta^{\mathrm{ann}}\right|(\mathrm{deg})$ & $P_{\mathrm{tim}}^{\text {bar }}$ & $H_{\mathrm{bar}}^{\text {patch }}$ & $\lambda_{\mathrm{obs}}(\mathrm{m})$ & Bar Orientation & $c_{\mathrm{obs}}(\mathrm{m} / \mathrm{d})$ \\
\hline Duck & 0.18 & 1.1 & 0.69 & 8.6 & 30 & $10 \%$ & $1-4$ & $79(12-179)$ & $32^{\text {ob }}$ & $<40^{\mathrm{c}}$ \\
Noordwijk & 0.20 & 1.6 & 0.76 & 5.7 & 45 & $14 \%$ & $3-9$ & $39(21-75)$ & $20^{\circ}$ up-current & $<22$ down-flow \\
\hline
\end{tabular}

${ }^{\mathrm{a}}$ Here, $d_{50}$ is the mean grain size, $\Delta z_{s}^{\mathrm{ann}}$ is the annual mean tidal range, $H_{\mathrm{rms}}^{\mathrm{ann}}, T_{p}^{\mathrm{ann}}$ and $\theta^{\mathrm{ann}}$ are the annual root mean square height, peak period and angle of incidence of the waves (respectively), measured at $18 \mathrm{~m}$ depth. Moreover, $P_{t i m}^{b a r}$ is the percentage of time with bar presence and $H_{b a r}^{p a t c h}$ is the number of bars per patch. The mean observed wavelength is $\lambda_{\text {obs }}$ (minimum and maximum values inside the parenthesis), the bar orientation is measured with respect to the shore-normal and $c_{\mathrm{obs}}$ is the observed migration rate, with the direction of movement.

${ }^{\mathrm{b}}$ Orientation with respect to the current not given.

${ }^{\mathrm{c}}$ Direction of migration unclear. 
Table 2. Results of the Models Reproducing Up-Current Oriented Surf Zone Bars ${ }^{\mathrm{a}}$

\begin{tabular}{lccccc}
\hline \multicolumn{1}{c}{ Reference } & Slope & $\theta$ & $\lambda(\mathrm{m})$ & $T(\mathrm{~h})$ & $c(\mathrm{~m} / \mathrm{d})$ \\
\hline Ribas et al. $[2003]$ & 0.020 & $15^{\circ}$ & 50 & 8 & 70 \\
Garnier et al. $[2006]$ & 0.050 & $25^{\circ}$ & 70 & 30 & 40 \\
\hline
\end{tabular}

${ }^{\mathrm{a}}$ Here, $\theta$ is the angle of wave incidence measured from the shore-normal at about $1 \mathrm{~m}$ depth in the first study and at $5 \mathrm{~m}$ depth in the second study. Also, $\lambda$ is the modeled wavelength, $T$ is the $e$-folding time for growth and $c$ is the migration rate.

neglected and that could have a potentially large impact on the $\mathcal{C}_{d a}$ profile is the turbulence-induced resuspension created by the rollers in the inner surf zone [Voulgaris and Collins, 2000; Butt et al., 2004]. Performing a more quantitative comparison between model results and field observations is the second essential step to test the bed-flow mechanism and obtain a better understanding of the underlying physical processes. Ribas et al. [2011] recently studied the effect of surface rollers on the profiles of the current and $\mathcal{C}_{d a}$ in the surf zone. They presented a new self-organization model that included the roller dynamics in the hydrodynamic equations and its effect in the sediment transport. The main focus of that paper was to calibrate the reference basic state of the model with wave height and current data of Egmond beach, the Netherlands. They also showed that rollers induce sediment resuspension in the inner surf zone so that $\mathcal{C}_{d a}$ becomes a decreasing function of the distance to the coast. Finally, they performed a few preliminary tests on nearshore bar formation (both crescentic bars and transverse finger bars), as an example of application of the new model. However, extensive model runs with different parameter values, the analysis of the obtained bar characteristics, the comparison with the observations and the explanation of the physical mechanisms were left for further research.

[8] The aims of the present contribution are to explain the characteristics of the surfzone transverse finger bars observed on open beaches and to reveal the physical mechanisms underlying their formation. Also, we want to provide fundamental information on why they are only observed in case of intermediate oblique waves. As one of our hypotheses is that sediment resuspension created by roller-induced turbulence is essential, we use the up-to-date self-organization model described by Ribas et al. [2011], which includes the roller dynamics (section 2). That model is also suitable because the wave height and longshore current profiles have already been calibrated with data from Egmond beach, Netherlands [Ribas et al., 2011], where offshore wave conditions are rather similar than those at Noordwijk. The methodology is based on linear stability analysis, which allows for a systematic exploration of the sensitivity of bar characteristics to the beach conditions. Moreover, this method reveals all the possible instability modes and it is a first step prior to performing non-linear temporal evolution. The results for transverse bar formation are explained in section 3 . The beach conditions measured at Noordwijk are used as model input, in order to perform a detailed and close comparison between transverse bar observations and model results. We also relate modeled bar characteristics to the hydrodynamic conditions in the inner surf zone to compare the present results with those of previous idealized models. The physical mechanism underlying the growth and migration of the bars is studied in detail in section 4. Specific attention is paid to the effect of the surface rollers on transverse bar formation. Section 5.1 contains the model-data comparison and explains several of the observed bar characteristics and their relation with the wave conditions. Section 5.2 addresses the robustness and limitations of the model. Finally, the conclusions are presented in section 6 .

\section{Model}

[9] The model used in this study is called MORFO62 [Ribas et al., 2011] and describes the feedbacks between depth-averaged mean currents, waves, rollers and an erodible bed in a nearshore zone with a straight shoreline. Compared with previous versions of the model, it has been extended by including the roller energy equation, the radiation stresses induced by rollers, and a novel term in the sediment transport formula that describes the sediment resuspension due to the turbulence created by the rollers. The domain represents a sea that is bounded by an alongshore uniform coast (Figure 2). A Cartesian coordinate system is chosen, in which the $y$ (or $x_{2}$ ) axis coincides with the rectilinear shoreline, the $x$ (or $x_{1}$ ) axis points in the seaward direction and the $z$ axis points upwards.

\subsection{Hydrodynamics}

[10] The large-scale fluid motions are governed by the wave- and depth-averaged momentum and mass conservation equations, assuming quasi-steady conditions $(\partial / \partial t=0)$,

$$
\begin{gathered}
v_{j} \frac{\partial v_{i}}{\partial x_{j}}=-g \frac{\partial z_{s}}{\partial x_{i}}-\frac{1}{\rho D} \frac{\partial}{\partial x_{j}}\left(\mathcal{S}_{i j}^{w}+\mathcal{S}_{i j}^{r}-\mathcal{S}_{i j}^{t}\right)-\frac{\tau_{b i}}{\rho D}, i, j=1,2, \\
\frac{\partial\left(D v_{j}\right)}{\partial x_{j}}=0, \quad j=1,2 .
\end{gathered}
$$

Here, repeated use of an index within a term means a summation over all values of this index, $\vec{v}=\left(v_{1}, v_{2}\right)$ is the waveaveraged mass transport velocity, $z_{s}$ is the mean free surface elevation and $D=z_{s}-z_{b}$ is the total depth, where $z=z_{b}$ is the sea bottom level. Furthermore, $g$ is the acceleration due to gravity and $\rho$ is the water density. The bed shear stresses in the $x$ - and $y$-direction are described by $\tau_{b 1}$ and $\tau_{b 2}$. The depthintegrated wave radiation stresses are denoted by $\mathcal{S}_{i j}^{w}$, while $\mathcal{S}_{i j}^{r}$ are the roller-induced radiation stresses, and $\mathcal{S}_{i j}^{t}$ are the Reynolds turbulent stresses.

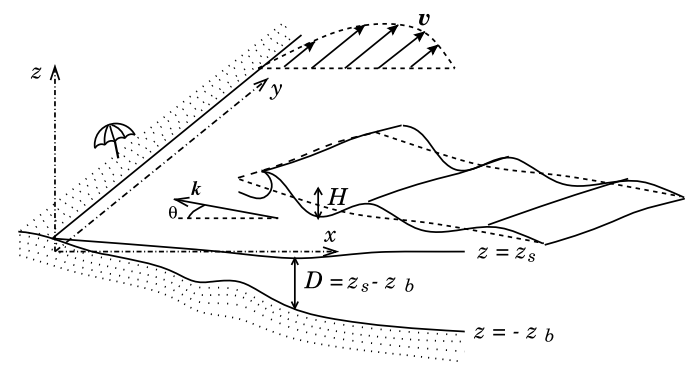

Figure 2. Sketch with the model frame of reference, including the coordinate system and some variables. 
Table 3. Default Parameter Setting of This Study and Range of Variation $^{\mathrm{a}}$

\begin{tabular}{llll}
\hline Symbol & \multicolumn{1}{c}{ Meaning } & Default & \multicolumn{1}{c}{ Range } \\
\hline$k_{a}$ & Apparent bed roughness & $0.035 \mathrm{~m}$ & $0.01-0.1 \mathrm{~m}$ \\
$M$ & Turbulence parameter & 1 & $0.1-10$ \\
$\gamma_{b}$ & Saturation ratio of $H / D$ & 0.475 & $0.30-0.75$ \\
$\beta_{\text {rol }}$ & Slope of wave/roller front & 0.05 & $0.04-0.1$ \\
$n_{\text {rol }}$ & Bore stirring parameter & 50 & $0-90$ \\
$H^{o f f}$ & Offshore RMS wave height & $0.75 \mathrm{~m}$ & $0.25-2.5 \mathrm{~m}$ \\
$T_{p}$ & Peak wave period & $5.5 \mathrm{~s}$ & $3.5-9.5 \mathrm{~s}$ \\
$\mid \theta^{\text {off }}$ & Offshore wave angle & $50^{\circ}$ & $0-80^{\circ}$ \\
\hline
\end{tabular}

${ }^{\mathrm{a}}$ The default values of $k_{a}, M, \gamma_{b}$ and $\beta_{\mathrm{rol}}$ are those best reproducing wave height and longshore current profiles at Egmond beach [Ribas et al., 2011]. The default values of the wave parameters are representative for average conditions measured at $18 \mathrm{~m}$ depth during the transverse bar events at Noordwijk [Ribas and Kroon, 2007].

[11] The present study considers oblique waves and therefore $\tau_{b i}$ are parameterized using a two-dimensional generalization of the formulation of Feddersen et al. [2000],

$$
\tau_{b i}=\rho c_{D} \frac{u_{\mathrm{rms}}}{\sqrt{2}} v_{i}\left(1.16^{2}+2 \frac{|\vec{v}|^{2}}{u_{\mathrm{rms}}^{2}}\right)^{1 / 2}, \quad i=1,2 .
$$

In this expression, $c_{D}$ is the drag coefficient and $u_{\mathrm{rms}}$ is the root mean square wave orbital velocity amplitude near the bed. We assume that $c_{D}$ varies with depth according to the Manning-Strickler law

$$
c_{D}=0.015\left(\frac{k_{a}}{D}\right)^{1 / 3} .
$$

Here, $k_{a}$ is the apparent bed roughness, assumed to be constant, and a range of realistic values is employed (Table 3 ). According to Ruessink et al. [2001], this empirical parameterization adequately represents the bed shear stresses at Egmond beach. The lateral turbulent mixing coefficient is linked to the dissipation of roller energy, $\nu_{t}=M\left(\mathcal{D}^{r} / \rho\right)^{1 / 3} D$, where $M$ is a parameter of $O(1)$ and $\mathcal{D}^{r}$ is the roller energy dissipation rate. The fluid velocities are imposed to vanish at the coastline and at the seaward boundary $(x \rightarrow \infty)$ and the free surface elevation is assumed to vanish offshore.

[12] Waves are assumed to have a narrow spectrum in frequency and wave vector. The wave heights are supposed to be random and follow the Rayleigh distribution, characterized by the root-mean square height, $H_{\text {rms }}$. The waves observed at Noordwijk beach obey these conditions. When waves approach the coast, their evolution is described using linear wave theory, which yields standard expressions for the wave radiation stresses $\mathcal{S}_{i j}^{w}$, the orbital velocity amplitude near bed, $u_{\mathrm{rms}}$, and the phase and group velocity magnitudes, $c$ and $c_{g}$. The dispersion relation for the absolute wave frequency (including the Doppler shift) is used to describe the refraction of the waves due to both topography and currents. Due to the wave number irrotationality, the dispersion relation can be written in terms of the wave phase, $\Phi$. From it, we define the absolute frequency, $\hat{\omega}=-\partial \Phi / \partial t$ and the wave vector components, $K_{i}=\partial \Phi / \partial x_{i}$. For brevity, we denote $H_{\mathrm{rms}}$ as $H$ and $|\vec{K}|$ as $K$ from now on. The peak wave period, $T_{p}$, follows from the absolute frequency and the angle of wave incidence, $\Theta$, is computed from the wave vector, $\vec{K}=K(\cos \Theta$, $\sin \Theta)$.
In the model, the angle $\Theta$ is measured anticlockwise with respect to the positive $x$ axis, but for the sake of simplicity, in the paper we will most often refer to the angle $\theta=\pi-\Theta$, which is the one between the wave rays and the negative $x$ axis (Figure 2). A more detailed description of the wave model is given by Ribas et al. [2011].

[13] The transformation of the wave energy is described with the equation

$$
\frac{\partial}{\partial x_{j}}\left(\left(v_{j}+c_{g j}\right) E\right)+\mathcal{S}_{j k}^{w} \frac{\partial v_{k}}{\partial x_{j}}=-\mathcal{D}^{w}, j, k=1,2,
$$

where $E=\rho g H^{2} / 8$ is the energy density of the random waves, and $c_{g j}$ are the components of the group velocity. The wave energy dissipation rate, $\mathcal{D}^{w}$, is parameterized using the formulation of Thornton and Guza [1983],

$$
\mathcal{D}^{w}=\frac{3 B^{3} \rho g \hat{\omega} H^{5}}{32 \sqrt{\pi} \gamma_{b}^{2} D^{3}}\left(1-\left(1+\left(\frac{H}{\gamma_{b} D}\right)^{2}\right)^{-2.5}\right),
$$

where $B$ is a parameter describing the type of breaking and $\gamma_{b}$ is the expected saturation value of $H / D$ for random wave heights. We assume $B=1$, so that the entire front of the wave is conceived to be covered with foam, consistent with the derivation of the roller equations [Lippmann et al., 1996]. The parameter $\gamma_{b}$ is assumed to be cross-shore uniform and we use a range of realistic values (see Thornton and Guza [1986] and Table 3).

[14] The energy dissipated by wave breaking feeds the surface rollers, i.e., the aerated mass of water located on the shoreward face of breaking waves. The roller energy balance is an extension of the one proposed by Reniers et al. [2004], which we have adapted to account for wave-current interactions,

$$
\frac{\partial}{\partial x_{j}}\left(2\left(v_{j}+c_{j}\right) E^{r}\right)+\mathcal{S}_{j k}^{r} \frac{\partial v_{k}}{\partial x_{j}}=-\mathcal{D}^{r}+\mathcal{D}^{w}, j, k=1,2 .
$$

Here, $E^{r}$ is the energy density of the rollers and $c_{j}$ are the components of the phase velocity. The roller-induced radiation stresses, $S_{i j}^{r}$, are computed following Svendsen [1984],

$$
S_{j k}^{r}=2 E^{r} \frac{K_{j} K_{k}}{|\vec{K}|^{2}}, j, k=1,2 .
$$

Finally, the roller energy dissipation rate, $D^{r}$, in equation (7) is modeled following Ruessink et al. [2001],

$$
\mathcal{D}^{r}=\frac{2 g E^{r} \sin \left(\beta_{\text {rol }}\right)}{c},
$$

where $\beta_{\text {rol }}$ is the angle of the wave/roller interface, usually assumed to be $\sin \left(\beta_{\text {rol }}\right) \leq 0.1$. Quasi-steady conditions have been also assumed in the wave propagation equations and wave conditions are prescribed at the offshore boundary $\left(H^{\text {off }}, T_{p}\right.$ and $\left.\theta^{\text {off }}\right)$.

\subsection{Bed Evolution and Sediment Transport}

[15] Conservation of sediment mass yields the bottom evolution equation,

$$
(1-p) \frac{\partial z_{b}}{\partial t}+\frac{\partial q_{j}}{\partial x_{j}}=0, \quad j=1,2
$$


where $p=0.4$ is the porosity of the bed and $q_{1}, q_{2}$ are the two components of the wave-averaged horizontal sediment transport (volume of sand per unit width and unit time, $\left.\mathrm{m}^{3} \mathrm{~m}^{-1} \mathrm{~s}^{-1}\right)$. A widely accepted formulation for $q_{i}$ in the nearshore is the Soulsby and van Rijn formula given by Soulsby [1997] (SvR-formula from now on). Their original expression has been extended to model the effect of a twodimensional flow and the preferred downslope transport of the sand,

$$
q_{i}=\mathcal{C}\left(v_{i}-\Gamma \frac{\partial h}{\partial x_{i}}\right), \quad i=1,2 .
$$

The function $\mathcal{C}$ describes the depth-integrated volumetric sediment concentration (also called sediment stirring). The second term inside the parenthesis of equation (11), where $\Gamma$ is called the bedslope coefficient and $h(x, y, t)$ stands for the perturbation of the sea bottom, accounts for the tendency of the system to smooth out such bottom perturbations if they do not cause positive feedback into the flow. In the SvR-formula (11), $\mathcal{C}$ reads

$$
\begin{array}{ll}
\mathcal{C}=A_{s}\left(u_{\text {stir }}-u_{\text {crit }}\right)^{2.4}, & \text { if } \quad u_{\text {stir }}>u_{\text {crit }}, \\
\mathcal{C}=0, & \text { otherwise. }
\end{array}
$$

Here, $A_{s}$ depends on the sediment properties (the grain size measured at the Dutch coast is $d_{50}=0.2 \mathrm{~mm}$ ) and $u_{\text {crit }}$ is the threshold flow intensity for sediment transport. The full expressions for the two latter quantities are given by Soulsby [1997]. The symbol $u_{\text {stir }}$ stands for the stirring velocity, which is assumed to originate from the shear stresses produced at the bed. In the original SvR-formula, only the shear stresses produced by the ordered wave orbital velocity and the depth-averaged currents were taken into account for estimating $u_{\text {stir. }}$. This SvR-formula was tested to be accurate in the shoaling domain, where depths are larger than $2 \mathrm{~m}$ [Soulsby, 1997]. Inside the inner surf zone, where $u_{\mathrm{rms}}$ and $|\vec{v}|$ are small, another process related with bore propagation and roller-induced turbulence has shown to be dominant and produce significant sediment resuspension [Komar, 1998; Voulgaris and Collins, 2000; Butt et al., 2004]. In the present study, the formulation for the stirring velocity $u_{\text {stir }}$ has been extended following Reniers et al. [2004] to account for rollerinduced resuspension,

$$
u_{\mathrm{stir}}=\left(|\vec{v}|^{2}+\frac{0.018}{c_{D}} u_{\mathrm{rms}}^{2}+n_{\mathrm{rol}} u_{\mathrm{rol}}^{2}\right)^{1 / 2}
$$

The two first terms of $u_{\text {stir }}$, which are the original ones in the SvR-formula, correspond to the stirring by currents and ordered wave motion, respectively, and the third term describes the stirring by the roller-induced turbulence. The symbol $u_{\text {rol }}$ represents the turbulence velocity of the vortices created after roller energy is dissipated and $n_{\text {rol }}$ is a constant parameter. We follow Roelvink and Stive [1989], who assumed that this extra $u_{\text {rol }}$ depends on the dissipation of roller energy,

$$
u_{\mathrm{rol}}=\left(\frac{\mathcal{D}^{r}}{\rho}\right)^{1 / 3}\left(e^{\left(D / H_{\mathrm{ms}}\right)}-1\right)^{-1 / 2},
$$

where the exponential function accounts for the decrease of the turbulent velocity from the surface to the bed. By varying the parameter $n_{\text {rol }}$ in equation (13), the strength of the sediment resuspension due to roller-induced turbulence can be varied. Values of $n_{\text {rol }}$ of about 50 give reasonable values of $\mathcal{C}$ of the order of $10^{-3} \mathrm{~m}$ in the inner surf zone [Roelvink and Stive, 1989].

[16] The bedslope coefficient $\Gamma$ in equation (11) is also assumed to depend on $u_{\text {stir }}$ [Calvete et al., 2005],

$$
\begin{array}{ll}
\Gamma=\gamma\left(u_{\text {stir }}-u_{\text {crit }}\right), & \text { if } \quad u_{\text {stir }}>u_{\text {crit }}, \\
\Gamma=0, & \text { otherwise, }
\end{array}
$$

where $\gamma$ is the bedslope parameter. The default value $\gamma=0.5$ yields bedslope coefficients similar in magnitude to those of the original Soulsby and van Rijn formulation [Soulsby, 1997]. We impose a fixed bed level $(h=0)$ at the seaward boundary and at the coastline, i.e., where water depth vanishes.

\subsection{Methodology}

[17] The governing equations and the parameterizations define a closed dynamical system for the unknowns $\vec{v}, z_{s}, E$, $E^{r}, \Phi$ and $z_{b}$. The stability analysis approach to the formation of bars by self-organization starts by defining an equilibrium (i.e., steady) and alongshore uniform basic state (without transverse bars). In this study, we use a reference profile, $z_{b}^{o}(x)$, measured at Noordwijk beach during the studied bar events (here, superscripts ${ }^{o}$ refer to the basic state variables). The offshore boundary is set at $18 \mathrm{~m}$ depth, the location of the wave buoy in front of Noordwijk. The basic state is characterized by the presence of a longshore current, $\vec{v}=\left(0, V^{o}(x)\right)$, and an elevation of the mean free surface, $z_{s}=z_{s}^{o}(x)$. This basic state represents a morphodynamic equilibrium only if the net cross-shore sediment transport vanishes. Thereby, an important assumption of the model is that the dynamics of the cross-shore profiles (2D) is significantly slower than the dynamics of the bars we intend to describe.

[18] The basic state of the model employed in the present study was calibrated with wave height and longshore current data from 9 days of observations in October 1998 at Egmond beach, the Netherlands [Ribas et al., 2011]. The values of $\gamma_{b}$ and $k_{a}$ were adjusted so that the profiles of $H^{o}$ and $V^{o}$ could be modeled with the minimum RMS error. The default values of $\beta_{\text {rol }}$ and $M$ were chosen for being standard widely used values, and they were subsequently shown to give nearly the smallest RMS errors of $H^{o}$ and $V^{o}$ [Ribas et al., 2011]. An example of measured and modeled $H^{o}$ and $V^{o}$ on 18 October 1998 at Egmond beach (when the wave conditions were similar to those of the default case of the present study) is shown in Figure 3. Using the calibrated values of the parameters, the reference wave height was modeled with an overall RMS error of $0.12 \mathrm{~m}$ and the longshore current was modeled with an error of $0.16 \mathrm{~m} \mathrm{~s}^{-1}$, similar to those obtained with other models [Ruessink et al., 2001]. The calibrated values of these parameters are the default values used in the present study (Table 3).

[19] After computing the basic state, stability analysis can be applied in a standard way. A small perturbation, assumed 


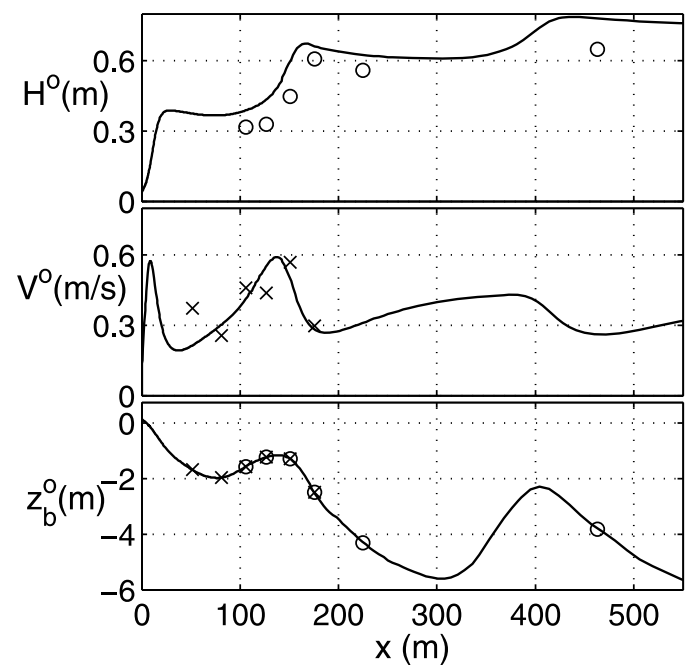

Figure 3. Example of modeled (solid line) and observed (circles and crosses) (top) RMS wave height, (middle) longshore current, and (bottom) the corresponding bed level at Egmond beach, the Netherlands. The hydrodynamic data was measured on 18 October 1998 at 20:00 UTC (wave conditions $H^{o f f}=0.82 \mathrm{~m}, T_{p}=7.7 \mathrm{~s}$ and $\left.\theta^{\text {off }}=49^{\circ}\right)$ and the bathymetry was also obtained on 18 October 1998. The positions of the pressure sensors (circles) and the current meters (crosses) are shown in Figure 3 (bottom).

to be exponential in time and periodic in the alongshore coordinate, is added to the basic state. In the case of the bed level this reads

$$
z_{b}(x, y, t)=z_{b}^{o}(x)+h(x, y, t), \text { where } h(x, y, t)=\mathfrak{R} e\left(e^{\omega t+i k y} \hat{h}(x)\right) .
$$

Here, $\hat{h}(x)$ stands for the cross-shore distribution of the perturbation of $z_{b}, \kappa$ is the alongshore wave number of these perturbations and $\omega$ is a complex growth rate. Expressions equivalent to equation (16) are used for the other six variables, where $u, v, \eta, e, e^{r}$ and $\phi$ correspond to the perturbations in $v_{1}, v_{2}, z_{s}, E, E^{r}$ and $\Phi$, respectively. By inserting all these expressions in the governing equations and linearizing with respect to the perturbations, we arrive at an eigenproblem. For each $\kappa$, different eigenvalues $\omega$ exist, which characterize the different growing modes, and the complex eigenfunctions are $\left(\hat{u}(x), \hat{v}(x), \hat{\eta}(x), \hat{e}(x), \hat{e}^{r}(x), \hat{\phi}(x), \hat{h}(x)\right)$. The $e$-folding growth rate of the evolving perturbations is given by $\Omega=\mathfrak{R} e(\omega)$, so that $\Omega>0$ means growth. In case of an unstable basic state, some perturbations with $\Omega>0$ are found. The growth rate curves show these positive $\Omega$ for different values of $\kappa$. Starting from arbitrary small amplitude initial conditions, the dynamics after some time will be dominated by the mode with largest growth rate, $\Omega_{M}$, and the corresponding $\kappa_{M}$. The $e$-folding growth time is given by $\tau_{M}=\Omega_{M}^{-1}$ and the migration speed by $c_{M}=-\Im m\left(\omega_{M}\right) / \kappa_{M}$. The alongshore wavelength of the corresponding bar patch is $\lambda_{M}=2 \pi / \kappa_{M}$ and its shape is given by $\mathfrak{R} e\left(e^{i \kappa_{M} y} \hat{h}(x)\right)$. The associated perturbations in the other variables are obtained in a similar way from $\hat{u}(x), \hat{v}(x), \hat{\eta}(x), \hat{e}(x), \hat{e}^{r}(x)$ and $\hat{\phi}(x)$. Given the uncertainties in the available sediment transport formulations, the function $\mathcal{C}$ in equation (11) is not perturbed.

\section{Results}

\subsection{Default Case Study}

[20] The default parameter setting of the present study (Table 3) reproduces the conditions measured at Noordwijk beach during the longest transverse bar event observed by Ribas and Kroon [2007], which lasted from 7 August to 5 October 2002 (Figure 1). The default bathymetric profile is representative of the one that was measured at $y=-500 \mathrm{~m}$ on 3 October 2002 during a large scale bathymetric survey, assuming mean sea level conditions. The result for the basic state of the default case shows that wave energy dissipation occurs above the two shore-parallel bars and at the inner surf zone (Figure 4, left). The two strongest peaks of the reference longshore current, $V^{o}$, are located over the inner shoreparallel bar crest, at $x=109 \mathrm{~m}$, and inside the inner surf zone, at $x=x_{\text {inn }}=6.9 \mathrm{~m}$. The values of some reference quantities at the latter position (plotted with a dashed line in Figure 4 (right)) are later used to interpret the results. Notice that, in the inner surf zone $(5<x<50 \mathrm{~m})$, the depthaveraged sediment concentration, $C^{\mathrm{o}} / D^{\mathrm{o}}$, decreases with increasing distance from the coast.

[21] The result of the linear stability analysis for the default case study reveals the presence of two different solutions (Figure 5). The largest growth rate is obtained for a mode that has $\kappa_{M}=0.124 \mathrm{~m}^{-1}$, hence the modeled dominant wavelength is $\lambda_{M}=51 \mathrm{~m}$, the $e$-folding growth time is $\tau_{M}=12 \mathrm{~h}$ and the migration rate is $c_{M}=32 \mathrm{~m} / \mathrm{d}$. As can be seen in Figure 5 (middle), the solution corresponding to this maximum consists of a patch of transverse finger bars showing an 'up-current orientation', similar to the features observed at Noordwijk by Ribas and Kroon [2007]. The gray scale pattern in this panel shows the shape of the topographic perturbation corresponding to this mode and the small arrows display the perturbations of the current. The angle between the bar crest orientation and the shore-normal, $\alpha_{\text {bar }}$, is $50^{\circ}$. As can be seen, offshore (onshore) directed current perturbations occur over the bar crests (troughs). Realize that the actual bed level is obtained by adding the bed level perturbation to the basic state bed level, $z_{b}^{o}$. The same applies to the flow: the longshore current $V^{o}$ must be added to the current perturbations to obtain the total flow. Notice that linear stability analysis does not provide the finite amplitude of the perturbations. As an example and according to this linear model, an arbitrary amplitude of the bed level perturbations of $10 \mathrm{~cm}$ would give velocity perturbations of some $10 \mathrm{~cm} \mathrm{~s}^{-1}$.

[22] A secondary maximum in the growth rate curve of Figure 5 is obtained with $\kappa_{M 2}=0.011 \mathrm{~m}^{-1}$, and it corresponds to the moulding of the shore-parallel inner bar into a crescentic bar (Figure 5, bottom). Its spacing is $\lambda_{M 2}=570 \mathrm{~m}$, in the range observed at Noordwijk by van Enckevort et al. [2004]. The $e$-folding growth time is $\tau_{M 2}=54 \mathrm{~h}$ and the migration rate is $c_{M 2}=66 \mathrm{~m} / \mathrm{d}$. Notice the difference in flow pattern between transverse and crescentic bars; in the latter case offshore directed current perturbations occur over the troughs. The focus of this study are transverse finger bars, hence the crescentic bar solution will be hardly discussed in the rest of the paper. 

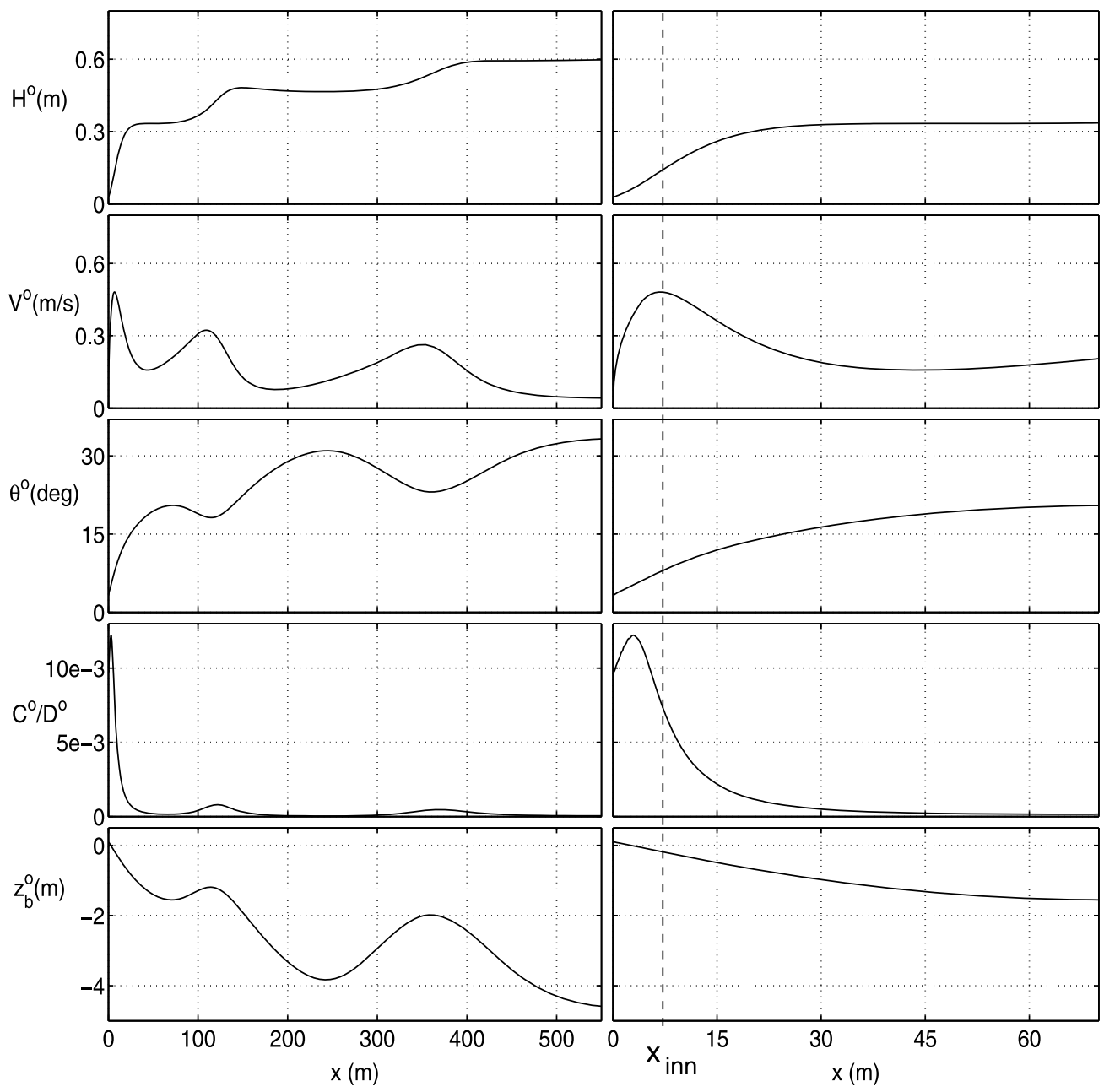

Figure 4. Reference basic state obtained with the default parameter setting, shown for (left) a cross-shore domain of $550 \mathrm{~m}$ and for (right) a cross-shore domain of $70 \mathrm{~m}$. Solution for (from top to bottom) the wave height, $H^{o}$, the longshore current, $V^{o}$, the RMS wave angle from the shore-normal, $\theta^{o}$, the depth-averaged volumetric sediment concentration, $\mathcal{C}^{o} / D^{o}$, and the bed level, $z_{b}^{o}$. The horizontal axis is the cross-shore position and the vertical dashed line points at the cross-shore position of the maximum longshore current, $x_{\text {inn }}$.

\subsection{Effect of the Offshore Wave Conditions on Model Results}

[23] The input wave conditions are varied based on those observed at Noordwijk from October 1998 to July 2004 (time period studied by Ribas and Kroon [2007]), which are shown in Figure 6. As can be seen in Figure 6 (top) for a fixed $H^{\text {off }}$ the value of $T_{p}$ ranges between a minimum value (dashed line in the figure) and some $9 \mathrm{~s}$. The averaged $T_{p}^{m}$ for each $H^{\text {off }}$ (solid line in the figure) is given by

$$
T_{p}^{m}=\alpha_{2}\left(H^{o f f}\right)^{2}+\alpha_{1} H^{o f f}+\alpha_{0},
$$

where $\alpha_{2}=0.025 \mathrm{~s} \mathrm{~m}^{-2}, \alpha_{1}=1.22 \mathrm{~s} \mathrm{~m}^{-1}$, and $\alpha_{0}=4.72 \mathrm{~s}$. Maximum values of $\left|\theta^{o f f}\right|$ also depend on $H^{\text {off }}$ (dashed lines in Figure 6 (bottom)). In the model runs, $H^{\text {off }}$ is varied from $0.25 \mathrm{~m}$ to $2.5 \mathrm{~m}$, with a step of $0.25 \mathrm{~m}, T_{p}$ is varied from $3.5 \mathrm{~s}$ to $9.5 \mathrm{~s}$, with a step of $1 \mathrm{~s}$, and $\left|\theta^{\circ f f}\right|$ is varied from $0^{\circ}$ to $80^{\circ}$, with a step of $10^{\circ}$.

[24] The variability in modeled up-current bar characteristics obtained when varying $H^{\text {off }}$ and $T_{p}$, while $\left|\theta^{\text {off }}\right|=50^{\circ}$, can be seen in Figure 7. In general, bar shape and dynamics hardly change when varying $T_{p}$ and keeping $H^{\text {off }}$ constant. The most noticeable effect is that $\Omega_{M}$ diminishes for the largest values of $T_{p}$, but bar growth occurs for all parameter values within the studied range. The offshore wave height, $H^{\text {off }}$, plays a stronger role. Bar formation is only obtained if $H^{o f f^{\prime}}>0.25 \mathrm{~m}$ and when $H^{\text {off }}$ is increased keeping $T_{p}$ constant, $\Omega_{M}$ and $c_{M}$ increase up to a maximum around $H^{o f f}=1-$ $2 \mathrm{~m}$, and they remain constant for larger $H^{\text {off }}$. If $H^{\text {off }}$ is changed while $T_{p}=T_{p}^{m}\left(H^{\text {off }}\right)$, following equation (17), the saturation of $\Omega_{M}$ is obtained for $H^{o f f}=0.75 \mathrm{~m}$ and $T_{p}=5.7 \mathrm{~s}$ (Figure 7, right). The wavelength $\lambda_{M}$ increases with increasing $H^{\text {off }}$. The bar crest orientation, $\alpha_{\text {bar }}$, remains constant when varying $H^{\text {off }}$ and decreases slightly when $T_{p}$ is larger.

[25] The strongest variability of up-current bar characteristics is found when changing $\left|\theta^{\text {off }}\right|$, as shown in Figure 8, where $H^{\text {off }}$ is also varied while $T_{p}=T_{p}^{m}\left(H^{\text {off }}\right)$. Only when $\left|\theta^{\text {off }}\right|>30^{\circ}$ the model simulates the growth of the up-current oriented bars. In that regime and for $H^{\text {off }}<1 \mathrm{~m}$, when $\left|\theta^{\text {off }}\right|$ is 


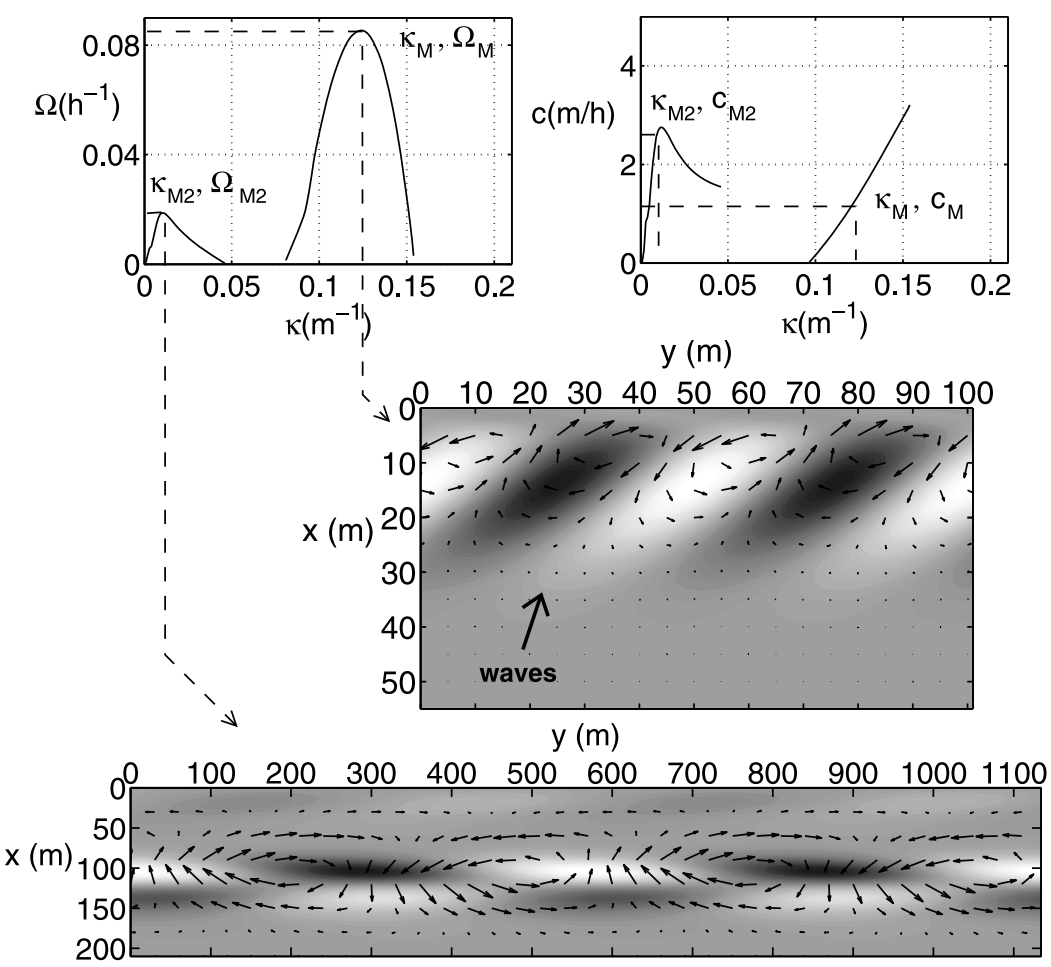

Figure 5. Results of the linear stability analysis of the default case. (top left) The growth rate curve (displaying $\Omega$ versus $\kappa$ ) and (top right) the migration rate curve (displaying $c$ versus $\kappa$ ) show the existence of two different modes. (middle) The topographic and current perturbations corresponding to the fastest growing mode, a patch of up-current oriented finger bars. (bottom) The perturbations corresponding to the secondary mode, a crescentic bar. In the latter plots, the shoreline $x=0$ is at the top. Waves approach the coast from the lower left corner so the induced mean longshore current is directed from left to right. In the topographic perturbations, white areas indicate crests and dark areas represent troughs. Small arrows are the deviations of the longshore current induced by the growing bars.

enlarged, $\Omega_{M}, \lambda_{M}$ and $c_{M}$ increase up to a certain value and then decrease again (Figure 8, right). This behavior is not obtained when $H^{\text {off }}>1 \mathrm{~m}$, the maximum in $\Omega_{M}$ not being reached for $\left|\theta^{\text {off }}\right|<80^{\circ}$. Notice that the regime $\left|\theta^{\text {off }}\right|>80^{\circ}$ is realistic only for $H^{\text {off }}<1.25 \mathrm{~m}$ (Figure 6 ). The shape of the bars is relatively robust when varying wave conditions. The modeled $\lambda_{M}$ changes $25 \%$ at most with respect to the default value. The bar crest orientation, $\left|\alpha_{\text {bar }}\right|$, varies $10 \%$ at most with respect to the default value (it increases for larger $\left|\theta^{o f f}\right|$ ) and $\alpha_{b a r}$ always changes its sign when the sign of $\theta^{\text {off }}$ is reversed (up-current orientation). When $\left|\theta^{\text {off }}\right|<30^{\circ}$, the only output of the model is the moulding of the inner shoreparallel bar into a crescentic bar, in agreement with previous research [Calvete et al., 2005].

[26] In order to interpret the obtained results and to compare them with those of previous idealized models, which used plane beach profiles [Ribas et al., 2003], the characteristics of the up-current oriented bars are subsequently related to the basic state values of some reference quantities at the inner surf zone: $\theta_{\text {inn }}, V_{\text {inn }}$ and $l_{\text {inn }}$. The symbols $\theta_{\text {inn }}$ and $V_{\text {inn }}$ refer to the values of $\theta^{o}$ and $V^{o}$ at the location of the innermost peak of the longshore current $\left(x_{\text {inn }}\right.$ position of Figure 4), and $l_{\text {inn }}$ is the distance from the shoreline to $x_{\text {inn }}$, i.e., a proxy of the surf zone width $\left(l_{\text {inn }}\right.$ and $x_{\text {inn }}$ differ due to wave setup). Bar growth only occurs if $\theta_{\text {inn }}>5^{\circ}$ (not shown), i.e., conditions met when $\theta^{\text {off }}>30^{\circ}$. The quantity that best correlates with $\Omega_{M}$ is $V_{\text {inn }}$ (circles in Figure 9 (right)). In agreement with the results of the previous idealized models [Ribas et al., 2003], $\lambda_{M}$ increases with increasing $l_{\text {inn }}$ and $c_{M}$ increases with increasing $V_{\mathrm{inn}}$ (Figure 9).

[27] The dependence of the up-current bar characteristics on the wave conditions can also be interpreted with the help of the inner surf zone parameters. The inner and outer shoreparallel bars of Noordwijk profile play an important role: they filter the waves in case of large $H^{\text {off }}$ such that $H^{o}$ always show similar values at the inner surf zone, thereby leading to

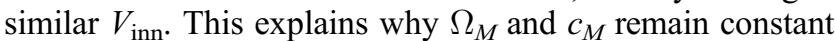
for $H^{\text {off }}>1-2 \mathrm{~m}$ (Figure 7). The decrease of $\Omega_{M}$ when increasing $T_{p}$ that can be seen in the same figure is due to the fact that long period waves experience a stronger refraction across the shoaling domain and the outer surf zone, so that they have smaller values of $\theta_{\mathrm{inn}}$ and $V_{\mathrm{inn}}$. The behavior of bar characteristics for large $\theta^{\text {off }}$ (i.e., $\theta^{\text {off }} \geq 80^{\circ}$ ) shown in Figure 8 can also be understood from the behavior of $V_{\text {inn. }}$. For low energy obliquely incident waves $\left(H^{\text {off }}<1 \mathrm{~m}\right)$, the dependence of $V_{\text {inn }}$ with $\theta^{o f f}$ shows a maximum for a certain $\theta^{\text {off }} \simeq 60-70^{\circ}$, because the strong refraction experienced by waves with $\theta^{\text {off }}>70^{\circ}$ implies a strong energy dispersion (thus waves reach the inner surf zone with lower $H^{\circ}$ and 


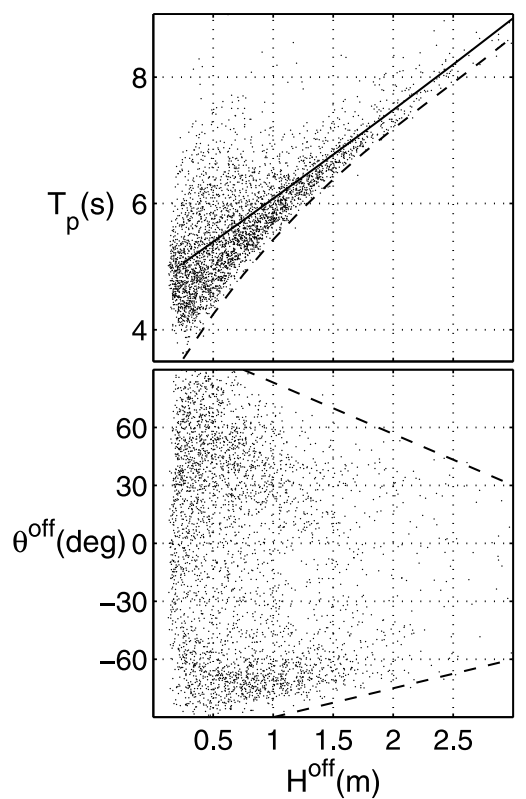

Figure 6. Values of $12 \mathrm{~h}$-averaged (top) $T_{p}$ versus $H^{\text {off }}$ and (bottom) $\theta^{\text {off }}$ versus $H^{\text {off }}$, measured at the Noordwijk buoy located at $18 \mathrm{~m}$ depth from October 1998 to July 2004. The solid line in Figure 6 (top) is the averaged $T_{p}^{m}$ for each $H^{\text {off }}$ (equation (17)), and the dashed line is the minimum $T_{p}$. The dashed lines in Figure 6 (bottom) point at maximum values of $\left|\theta^{\text {off }}\right|$ for each $H^{\text {off }}$.

$\left.V_{\text {inn }}\right)$. However, when $H^{\text {off }} \geq 1 \mathrm{~m}$ the energy dispersion effect that occurs for $\theta^{\text {off }}>70^{\circ}$ is unnoticeable due to the filtering effect of the shore-parallel bars, and $\Omega_{M}$ keeps increasing for $\theta^{\text {off }}$ up to $80^{\circ}$.

\subsection{Effect of the Underlying Profiles on Model Results}

[28] In order to model beach morphodynamics an initial bathymetry is always needed. Bathymetries are measured very scarcely in the field (a few times a year), whereas nearshore bar characteristics have proved to be very sensitive to the details of the underlying profile [Calvete et al., 2007]. Therefore, the sensitivity of the modeled up-current bar properties to varying some of the profile characteristics is analyzed, keeping the wave conditions equal to their default values. Since the transverse finger bars grow in the inner surf zone, the following bathymetric parameters are varied: the slope of the inner surf zone, $\beta_{\text {inn }}$ (i.e., the slope of first $50 \mathrm{~m}$ of the profile), the bed level of the inner bar crest, $z_{\text {bar }}$, and the bed level of the inner bar trough, $z_{\text {tro }}$. These parameters have an impact into the up-current bar characteristics of the same order than the wave parameters (Figure 10). The relationships between bar characteristics obtained when varying the bathymetric parameters and inner surf zone parameters (i.e., $\theta_{\text {inn }}, V_{\text {inn }}$ and $l_{\text {inn }}$ ) turn out to be very similar to those described in the previous section (compare circles and crosses in Figure 9).

[29] Slight increases in $\beta_{\text {inn }}$ lead to larger growth and migration rates (Figure 10, left) because narrower, more peaked longshore current profiles are created at the inner surf zone (i.e., larger $\left.V_{\text {inn }}\right)$. The shape of the bar patch $\left(\lambda_{M}\right.$ and $\alpha_{b a r}$ ) hardly changes when varying $\beta_{\mathrm{inn}}$. When $z_{\mathrm{bar}}$ and $z_{\text {tro }}$ are increased with respect to their default values, $\Omega_{M}$ and
$c_{M}$ decrease (Figure 10, middle and right). In the extreme case of $z_{\mathrm{bar}}=-0.6 \mathrm{~m}$, most of the waves break before reaching the inner bar crest and the longshore current becomes very weak in the inner surf zone (i.e., small $V_{\text {inn }}$ ). In the extreme case of $z_{\text {tro }}=-1 \mathrm{~m}$, the profile becomes terraced and $\beta_{\text {inn }}$ diminishes, leading to smaller growth rates. The modeled $\lambda_{M}$ and $\alpha_{\text {bar }}$ changes up to $40 \%$ and $10 \%$ with respect to their default values (respectively) when changing $z_{\mathrm{bar}}$ and $z_{\text {tro }}$, but no qualitative changes are found.

\subsection{Role of the Surface Rollers and Other Physical Processes}

[30] Given the limited experimental knowledge regarding turbulence stirring due to roller propagation, the value of the roller stirring parameter $n_{\text {rol }}$ in equation (13) is varied over a wide range (Figure 11). The characteristics of up-current bars depend significantly on this parameter. The formation of up-current oriented bars only occurs when $n_{\text {rol }}>20$, and the growth and migration rates increase with increasing $n_{\text {rol }}$ (due to the corresponding gain in sediment concentration, $C^{o}$ ). For large values of $n_{\text {rol }}$, bar characteristics become less dependent on this parameter. When $n_{\text {rol }}<20$ (i.e., tending to the original Soulsby and van Rijn formula), the model only reproduces the moulding of the inner shore-parallel bar into a crescentic bar. Thereby, a clear outcome of this analysis is that including the stirring by roller-induced turbulence is essential in order to model up-current oriented bars like those observed at Noordwijk (in the framework of our modeling approach).

[31] The other model parameter related to the presence of surface rollers, the slope of the roller surface, $\beta_{\text {rol }}$, is also varied and bar characteristics remain unchanged. In order to check the robustness of bar characteristics, the sensitivity of model results to varying the value of three other model parameters (the apparent bed roughness, $k_{a}$, the turbulence parameter, $M$, and the saturation ratio, $\gamma_{b}$ ) is subsequently checked. The formation of transverse bars occurs for most of the range of realistic values of these parameters. Increasing $k_{a}$ and $M$ causes the longshore current magnitude to decrease and results in smaller growth and migration rates of the transverse bars. Increasing $\gamma_{b}$ allows for greater values of $H^{o}$ and hence of $V_{\mathrm{inn}}$, and this increases the values of $\Omega_{M}$ and $c_{M}$. The shape of the modeled bars is robust under changes of model parameters (provided that $n_{\text {rol }}>20$ ). Bar orientation is always up-current with $\alpha_{\text {bar }}$ varying $10 \%$ at most and the values of $\lambda_{M}$ deviate some $25 \%$ from the default value.

\section{Physical Mechanisms Behind Up-Current Bar Development}

[32] Ribas et al. [2003] were the first to show that the socalled bed-flow mechanism provides a physical explanation for the development of up-current oriented bars. They did this in the framework of their highly idealized model (e.g., uniform beach slope, no random wave heights, no rollers, idealized wave refraction, simple algebraic formula for depth-integrated concentration). As the present model accounts for more detailed physics and uses cross-shore bathymetric profiles showing two shore-parallel bars, it is important to investigate whether these extensions affect the development mechanisms of the bars. Thereby, the interaction between the flow and the morphology has been studied in detail by first understanding 


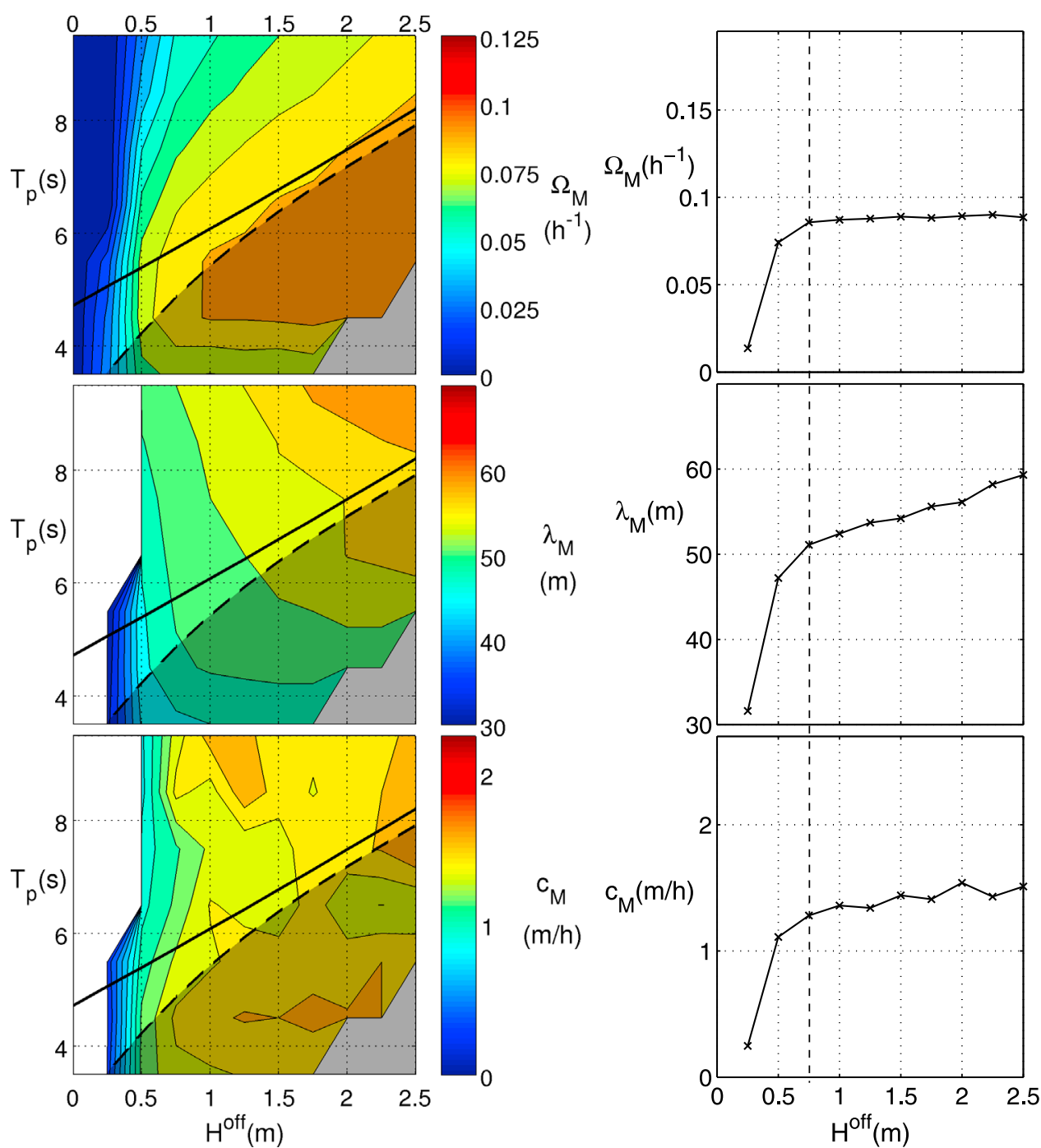

Figure 7. Sensitivity of the characteristics of modeled up-current oriented bars to varying the offshore wave height, $H^{\text {off }}$, and period, $T_{p}$, while $\theta^{\text {off }}=50^{\circ}$. Sensitivity of (top left) $\Omega_{M}$, (middle left) $\lambda_{M}$, and (bottom left) $c_{M}$ when $H^{\text {off }}$ and $T_{p}$ are varied. The solid lines indicate the averaged $T_{p}^{m}$ for each $H^{\text {off }}$ and the shaded area indicates unrealistic values of $H^{\text {off }}-T_{p}$ (Figure 6). Sensitivity of (top right) $\Omega_{M}$, (middle right) $\lambda_{M}$, and (bottom right) $c_{M}$ when $H^{\text {off }}$ is varied while $T_{p}=T_{p}^{m}\left(H^{\text {off }}\right)$, following equation (17). The vertical dashed line indicates the default $H^{\text {off }}$ value.

the accretion/erosion pattern created by a particular flow, and second analyzing the flow caused by a given topography.

[33] First, we follow Ribas et al. [2003] to derive a simplified bed evolution equation. Substituting $\vec{\nabla} \cdot \vec{v}$ from the water mass conservation equation (2) into the bottom evolution equation (10), linearizing with respect to the perturbations, and taking into account that $\left|\frac{C^{o}}{D^{o}} \frac{\partial(\eta-h)}{\partial t}\right| \ll\left|\frac{\partial h}{\partial t}\right|$, the following equation is obtained,

$$
\begin{aligned}
\frac{\partial h}{\partial t}= & -\frac{D^{o}}{(1-p)} \frac{d}{d x}\left(\frac{C^{o}}{D^{o}}\right) u+\frac{V^{o} C^{o}}{D^{o}(1-p)} \frac{\partial(\eta-h)}{\partial y} \\
& +\frac{1}{(1-p)} \vec{\nabla}\left(C^{o} \Gamma^{o} \vec{\nabla} h\right) .
\end{aligned}
$$

Here, $C^{o}$ is the depth-integrated sediment concentration (equation (12)) and $\Gamma^{o}$ is the bed slope coefficient (equation (15)).
The superscript ${ }^{o}$ refers to basic state variables and $h, \eta$ and $u$ are the perturbations of the bed level, the water level and the cross-shore component of the velocity, respectively. The three terms in the RHS of equation (18) are named $T_{1}, T_{2}$ and $T_{3}$ for further analysis. Subsequently, we follow Garnier et al. [2006] and Vis-Star et al. [2008] to perform a global analysis of the bed evolution equation (18). The instantaneous global growth rate, $\Omega^{\text {glo }}$, and migration rate, $c^{\text {glo }}$, of a solution can be defined as

$$
\Omega^{\text {glo }}=\frac{\left\langle h \frac{\partial h}{\partial t}\right\rangle}{\left\langle h^{2}\right\rangle}, \quad \text { and } \quad c^{\text {glo }}=-\frac{\left\langle\frac{\partial h}{\partial y} \frac{\partial h}{\partial t}\right\rangle}{\left\langle\left(\frac{\partial h}{\partial y}\right)^{2}\right\rangle},
$$

where $\langle f\rangle$ denote an average over the computational domain. It can be proved that, in a linear stability analysis where the 


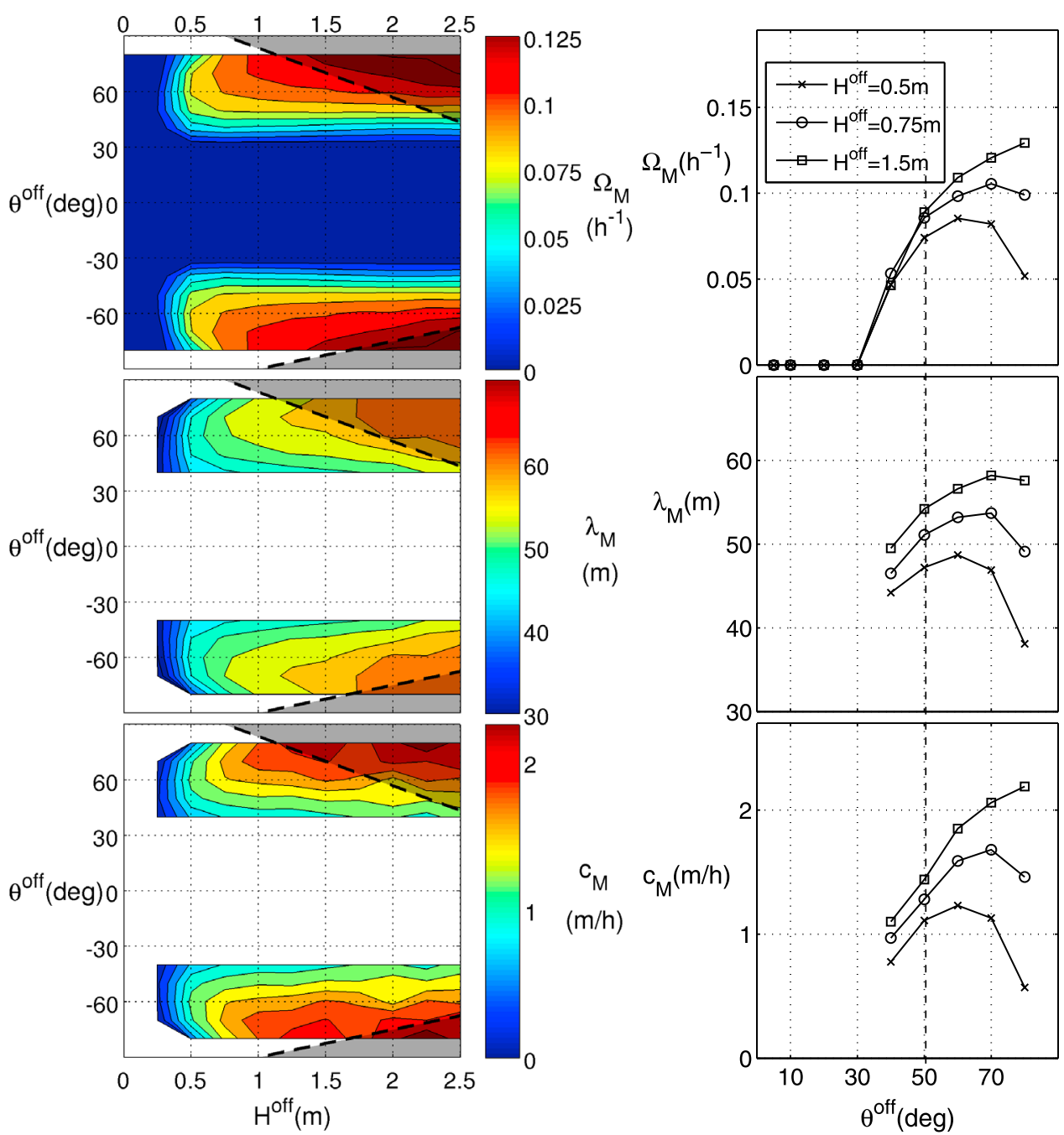

Figure 8. Sensitivity of the characteristics of modeled up-current oriented bars to varying the offshore wave height, $H^{\text {off }}$, and angle, $\theta^{\text {off }}$, while $T_{p}=T_{p}^{m}\left(H^{\text {off }}\right)$, following equation (17). Sensitivity of (top left) $\Omega_{M}$, (middle left) $\lambda_{M}$, and (bottom left) $c_{M}$ when $H^{\text {off }}$ (and $T_{p}$ ) are varied. The shaded areas indicate unrealistic values of $H^{\text {off }}-\theta^{\text {off }}$ (Figure 6). Sensitivity of (top right) $\Omega_{M}$, (middle right) $\lambda_{M}$, and (bottom right) $c_{M}$ when $\theta^{o f f}$ is varied. The curves correspond to different values of $H^{o f f}$ and the vertical dashed line indicates the default $\theta^{\text {off }}$ value.
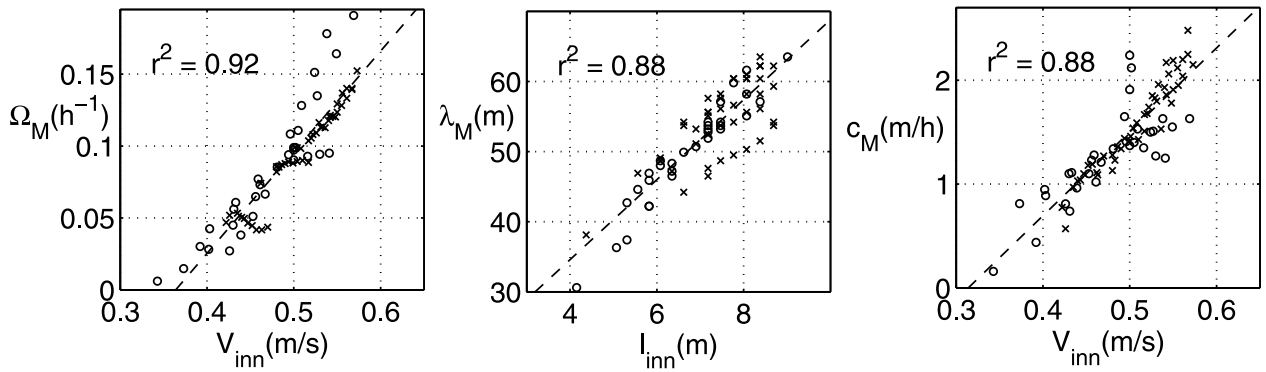

Figure 9. Dependence of the modeled up-current oriented bar characteristics on the inner surf zone values $V_{\text {inn }}$ and $l_{\text {inn }}$ : (left) $\Omega_{M}$ versus $V_{\text {inn }}$, (middle) $\lambda_{M}$ versus $l_{\text {inn }}$, (right) and $c_{M}$ versus $V_{\text {inn }}$. The results obtained when varying the offshore wave conditions are displayed by circles and those obtained when varying the bathymetric parameters are plotted as crosses. The least squares fit lines are plotted in dashes and $r^{2}$ is the corresponding correlation coefficient. 

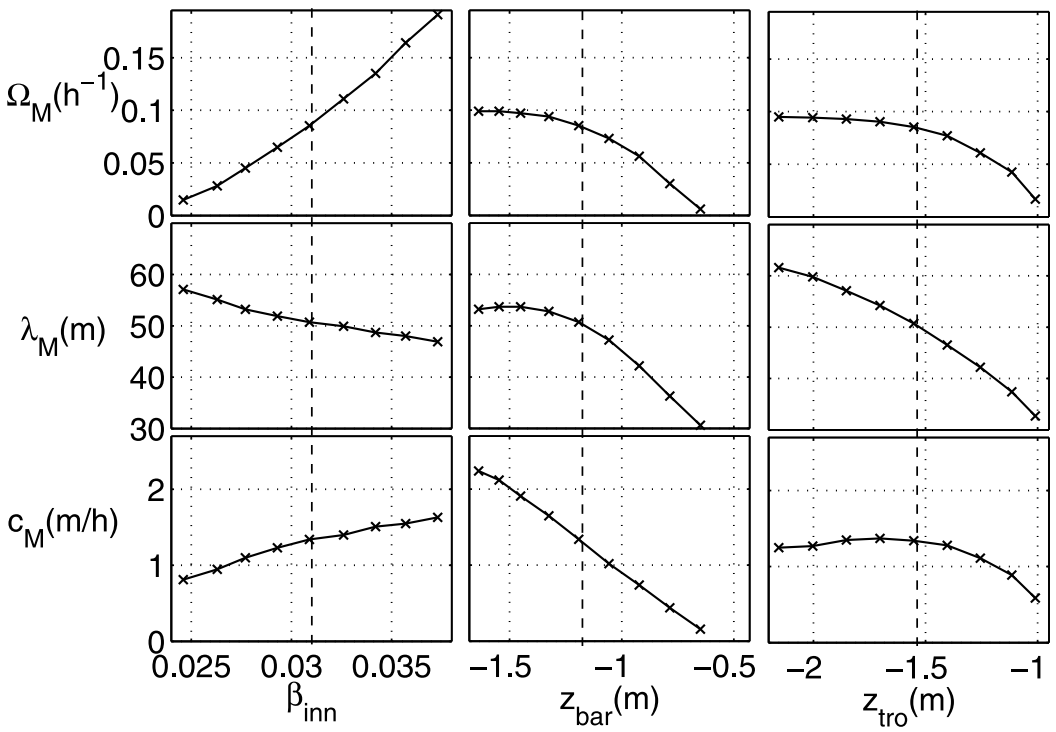

Figure 10. Sensitivity of (top) $\Omega_{M}$, (middle) $\lambda_{M}$, and (bottom) $c_{M}$ of modeled up-current oriented bars to changing three bathymetric parameters: (from left to right) the inner surf zone slope, $\beta_{\text {inn }}$, the bed level of the inner bar crest $z_{\text {bar }}$, and the bed level of the inner bar trough, $z_{\text {tro }}$. The vertical dashed lines show the values for the default bathymetric profile.

perturbations consist of a single alongshore wave, the obtained growth and migration rates, $\Omega$ and $c$ defined in section 2.3 , are equal to the global ones defined in equation (19). In order to investigate the contribution of the three terms $T_{n}(n=1,2,3)$ of the RHS of equation (18) to the global growth and migration of the bars we substitute these terms for the $\frac{\partial h}{\partial t}$ in equations (19). The values of the $\Omega_{n}^{\text {glo }}$ and $c_{n}^{\text {glo }}$, corresponding to these three terms $T_{n}$, have been calculated for the up-current bar pattern obtained in the default case and are shown in Table 4. The

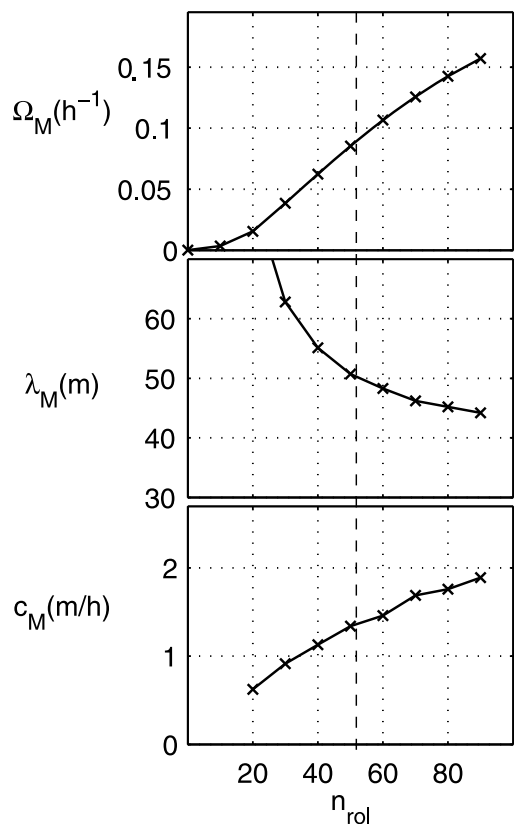

Figure 11. Results of (top) $\Omega_{M}$, (middle) $\lambda_{M}$, and (bottom) $c_{M}$ when the roller-induced turbulence stirring parameter $\left(n_{\text {rol }}\right.$ in equation (13)) is varied. The vertical dashed line indicates the default $n_{\text {rol }}$ value. interpretation of the result must be done by recalling the following properties of the solutions. First, in the area where bars grow $(5<x<50 \mathrm{~m})$, the depth-averaged sediment concentration, $C_{d a}^{o}=C^{o} / D^{o}$, decreases with increasing distance from the coast (Figure 4). Second, the obtained overall offshore deflection of the current over the crests (Figure 5) implies a positive correlation between $u$ and $h$. Third, the maximum in $u$ is located upstream of the maximum in $h$. This behavior of $C_{d a}^{o}$ and $u$ shows that the term $T_{1}$ provides bar growth and contributes to up-drift migration. Furthermore, in the region of the bars the maximum in $\frac{\partial(\eta-h)}{\partial y}$ is located downstream of the maximum in $h$ and they are approximately out of phase $(\eta \ll h$ in that region). Consequently $T_{2}$ is the agent that causes the down-drift migration. Finally, the term $T_{3}$ is a diffusive term that causes damping of the bottom perturbations. Applying this global analysis to other parameter values gives qualitatively similar results. Thus, as in the simplified model by Ribas et al. [2003], the mechanism responsible for bar growth is a combination of the offshore deflection of the current over the bars and the decrease of the depth-averaged sediment concentration in the seaward direction.

[34] Finally, we must explain why the longshore current deflects offshore over the bar crests, a phenomenon that indeed occurs only if bars are up-current oriented. There are two known hydrodynamic mechanisms that can cause an offshore current deflection over up-current oriented bars. The first was described by Trowbridge [1995] and is based

Table 4. Contribution of the Three Terms of the RHS of Equation (18) to the Global Growth and Migration

\begin{tabular}{lcc}
\hline \multicolumn{1}{c}{$\operatorname{Term} T_{n}$} & $\Omega_{n}^{\text {glo }}\left(\mathrm{h}^{-1}\right)$ & $c_{n}^{\text {glo }}(\mathrm{m} / \mathrm{h})$ \\
\hline$T_{1}=-\frac{D^{o}}{(1-p)} \frac{d}{d x}\left(\frac{C^{o}}{D^{o}}\right) u$ & +0.340 & -141 \\
$T_{2}=\frac{V^{o} C^{o}}{D^{o}(1-p)} \frac{\partial d}{\partial y}$ & -0.092 & +173 \\
$T_{3}=\frac{1}{(1-p)} \vec{\nabla}\left(C^{o} \Gamma^{o} \vec{\nabla} h\right)$ & -0.161 & 0 \\
\hline
\end{tabular}



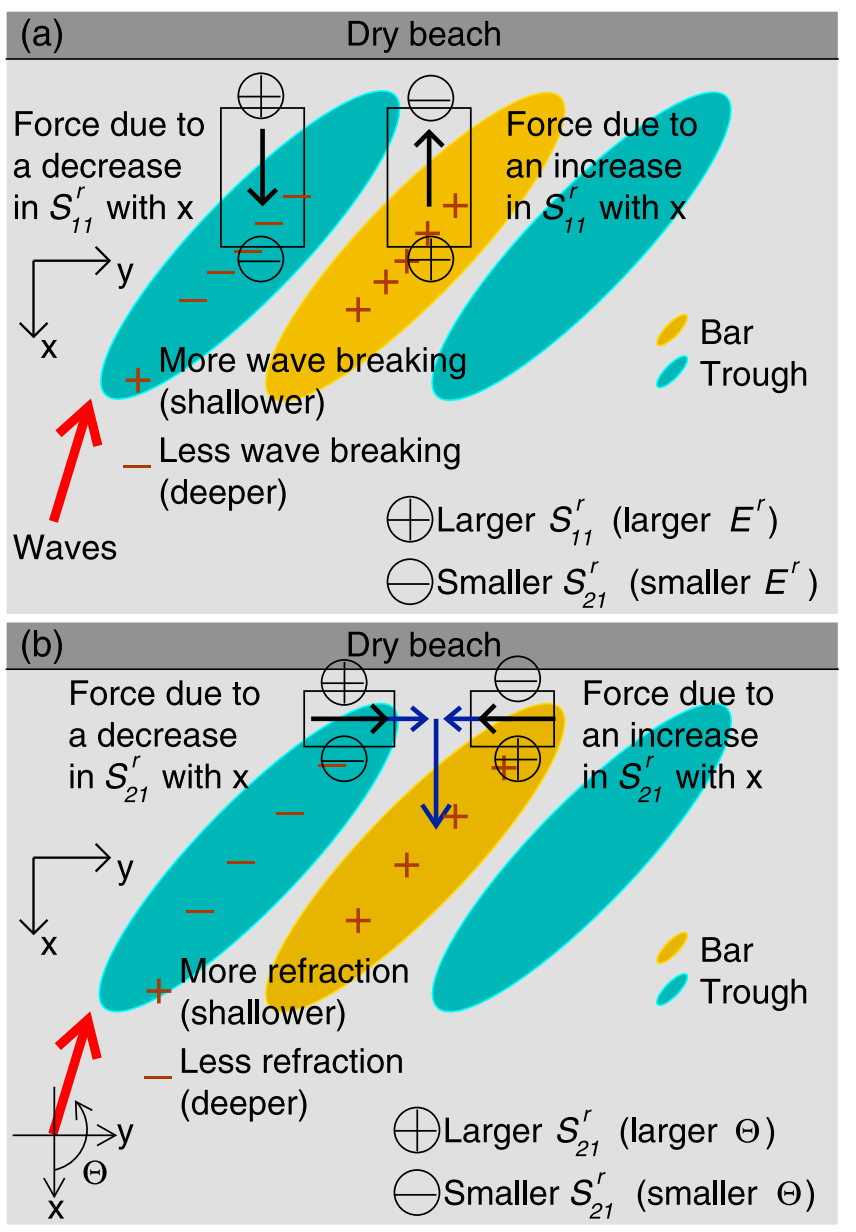

Figure 12. Schematic illustration of the effect of the roller radiation stresses into up-current bar formation. (a) The main effect of $S_{11}^{r}$ into the cross-shore momentum balance is to damp the occurrence of $u>0$ over the bar crests. (b) The main effect of $S_{21}^{r}$ into the alongshore momentum balance is to create the feeders that enhance the formation of $u>0$ over the bar crests.

on water mass conservation. When the longshore current flows over an elongated, up-current oriented bar, the crossbar velocity component becomes larger due to water mass conservation because the depth decreases. Moreover, since bar length is much larger than bar width, the alongbar component hardly changes, giving an offshore current deflection. The second mechanism was first described by Zimmerman [1981] and is related to the frictional torques created by changes in depth. When water columns move from the trough to the crest of an up-current oriented bar, they experience a clockwise rotation (and thus an offshore deflection) because friction is larger over the crest than at the trough. Note that both mechanisms would give an onshore deflection of the current over down-current oriented bars. To test whether the mass conservation mechanism plays an important role in our model we have performed a model run in which we have switched off all radiation stresses and friction terms in the momentum equations governing the perturbations, thereby effectively causing the perturbed flow to be irrotational. The result is that no growing solutions are obtained, and thus the mechanism of Trowbridge [1995] in itself does not provide the explanation for the emergence of the transverse finger bars. We have also tested the relevance of the frictional torques mechanism by performing a run in which only the radiation stresses have been switched off. This yields the formation of up-current oriented bars, with a growth rate that is 4 times larger than that of the default case. Thereby, frictional torques play an important role in the formation of up-current bars.

[35] However, the forces due to radiation stresses are also important, as they cause a reduction of the growth rate. To discriminate between wave and roller radiation stresses, we have performed two runs in which we have switched them off separately. It turns out that the roller radiation stresses have a stronger impact on reducing bar growth than wave radiation stresses. The following process, which occurs when including roller radiation stresses, causes the mechanism for bar formation to become less effective. Waves break when they reach the seaward end of the bar, thereby resulting in an increase of the roller energy density over the bar (compared with the situation without a bar). This roller energy dissipates when waves propagate further onshore (Figure 12a shows a sketch). Thus, the component $S_{11}^{r}$ of the roller radiation stress tensor $\left(S_{11}^{r}=2 E^{r} \cos ^{2} \Theta\right)$, decreases from the center of the bar to the shoreline, with the corresponding cross-shore force $F_{x} \propto-d S_{11}^{r} / d x$ pointing shoreward. The opposite happens at the troughs, where a seaward directed force is produced (forces are thick black arrows in Figure 12a). These forces would generate onshore (offshore) flow perturbations over the crests (troughs) and thus cause damping of the bars. There is, however, a second process also related with roller radiation stresses that contributes to bar growth. Waves experience a stronger refraction when propagating over a bar, compared with the situation without a bar, so that $\Theta$ is larger at the shoreward end of the bar and decreases rapidly up to the shoreline (see Figure $12 \mathrm{~b}$ for a sketch). Thereby, the component $S_{21}^{r}$ of the roller radiation stress tensor $\left(S_{21}^{r}=E^{r} \sin (2 \Theta)\right)$ also decreases and the corresponding alongshore force is directed to the negative $y$ axis, $F_{y} \propto-d S_{21}^{r} / d x$, creating an alongshore flow in that direction. The opposite happens at the seaward end of the trough, where a force is produced that points in the positive $y$ direction and creates an alongshore flow in that direction. These two feeders converge and generate a seaward directed flow over the bar due to continuity, contributing to bar growth (see currents in blue arrows in Figure 12b). The importance of this mechanism is proved by the fact that if $S_{11}^{r}$ is included but $S_{21}^{r}$ is not, up-current bars do not grow. In order to know whether the frictional torques are still playing an important role in the default model configuration, we have made one run in which radiation stresses have been included but friction terms have been switched off. With such configuration no growing solution is obtained either.

[36] We thus conclude that both the offshore current deflection produced by frictional torques and the $S_{21}^{r}$ induced feeders produced by changes in wave refraction contribute in an essential way to the establishment of the offshore current deflection over the bar crests needed for bar formation. When the offshore angle of wave incidence is smaller than $30^{\circ}$, these two mechanisms become less effective, while the $S_{11}^{r}$-induced forces become more effective, 
Table 5. Range of Values of Wavelength, Crest Orientation and Migration Rate From the Model and From the Observations at Noordwijk

\begin{tabular}{lcc}
\hline \multicolumn{1}{c}{ Quantity } & Model & Data $^{\text {a }}$ \\
\hline Wavelength (m) & $30-70$ & $21-75$ \\
Crest orientation (deg) & $45-55$ & $0-43$ \\
Migration rate (m/d) & $0-50$ & $0-22$ \\
\hline
\end{tabular}

${ }^{\mathrm{a}}$ Migration rates in the data column are daily averaged.

creating an onshore directed flow over the bars that inhibits bar growth.

\section{Discussion}

\subsection{Comparison of Model Results and Field Observations}

[37] The gross characteristics of the transverse bars observed at Noordwijk beach [Ribas and Kroon, 2007] are well reproduced by the present model. The result of the default case is in good agreement with the transverse bar patch observed in August and September 2002, which had a wavelength $\lambda_{\text {obs }}=46 \mathrm{~m}$, bar crests with an up-current orientation of $32^{\circ}$, daily migration rates varying from -8 to $22 \mathrm{~m} / \mathrm{d}$ and time for bar formation of about 2 days. When all the model results are included in the analysis, the magnitude and variability of the transverse bar characteristics observed from October 1998 to October 2004 is also explained (Table 5). Bar shape compares quite well, especially the wavelength and the fact that bars are always oriented up-current. However, the model results show quite constant and large angles of orientation whereas the angles in the field were smaller and more variable. Notice that measuring bar orientation on the field was somewhat qualitative because only a certain part of the bars was visible (the shallower part, where waves break). Modeled migration rates are about 2 times larger than the observed ones. Possible explanations for this discrepancy are postponed until the last paragraph of this section. In general, the growth time was not provided by Ribas and Kroon [2007] since the resolution of the video observations did not allow for its quantification. However, its order of magnitude could be estimated by the fact that wave conditions allowing bar formation commonly persisted for at least one day before a patch of transverse bars was detected for the first time, consistent with the modeled growth time.

[38] Linear stability analysis is not designed to make accurate predictions, but to give insight into the basic physics underlying the phenomenon. Therefore, it is more interesting to compare the modeled relationships and tendencies than the numbers themselves. Both in the model and in the observations, the wavelength of the bars shows a positive correlation with proxies of the width of the inner surf zone and the migration rate correlates with proxies of the longshore current (see Figure 9 and Ribas and Kroon [2007]). From our model results, we can also find an explanation of why bar patches observed at Noordwijk coexisted with intermediate waves $\left(0.25 \mathrm{~m}<H^{\text {off }}<1.5 \mathrm{~m}\right)$ and large angles of incidence with respect to the shorenormal $\left(\theta^{o f f}>25^{\circ}\right)$. This property is shown in Figure 13 (top and middle), which display the frequency of occurrence of a certain $H^{o f f}$ and $\theta^{o f f}$ at the Noordwijk buoy during the 6-year period studied by Ribas and Kroon [2007] (Figure 13, top) and the frequency of occurrence of $H^{\text {off }}$ and $\theta^{\text {off }}$ during the periods when bar patches were observed (14\% of the time, Figure 13, middle). These measurements are consistent with our results: bar growth only occurs in the model if wave incidence is shore-oblique, $\theta^{\text {off }}>30^{\circ}$, and if wave height is larger than $0.25 \mathrm{~m}$ (Figures 7 and 8). The fact that transverse bars were not observed to coexist with $H^{\text {off }}>1.5 \mathrm{~m}$, although modeled bars grow even faster for such heights, could be due to the fact that these wave heights are uncommon at Noordwijk. In order to verify the latter, a more quantitative comparison between the wave conditions measured during the presence of bars and the modeled wave conditions for bar growth is needed. This is done in Figure 13 (bottom), which displays the frequency measured during the entire study period (i.e., Figure 13, top) multiplied by the modeled growth rate (i.e., Figure 8, top left). As can be seen, the resulting distribution compares fairly well with that of the frequency measured during the presence of the bars (i.e., Figure 13,

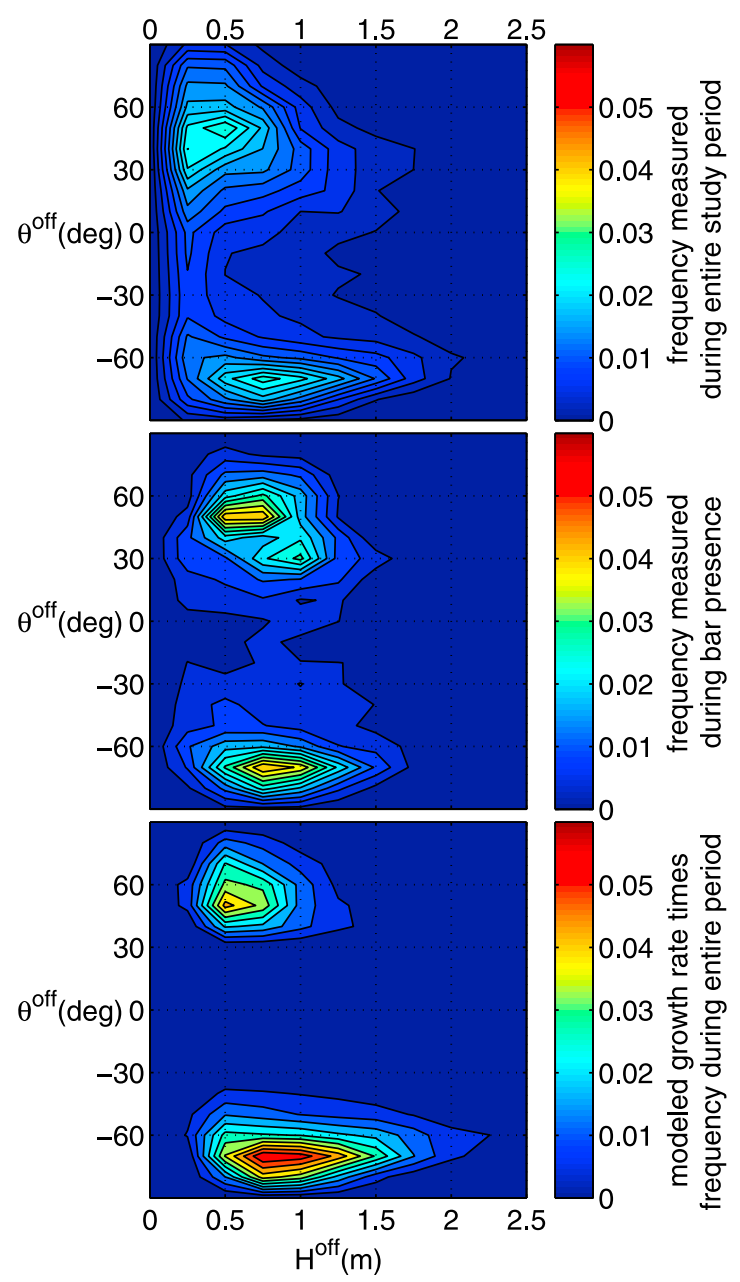

Figure 13. (top) Frequency of occurrence of $H^{\text {off }}$ and $\theta^{\text {off }}$ measured at the Noordwijk buoy (18 $\mathrm{m}$ depth) during the entire period studied by Ribas and Kroon [2007] (from Oct-98 to Oct-04). (middle) Frequency measured during the time with finger bar patches. (bottom) Modeled growth rate (Figure 8, top left) multiplied by frequency measured during the entire study period (Figure 13, top). 
middle). Finally, the reason why transverse bar patches at Duck (USA) occurred less frequently and had a smaller number of bars than in Noordwijk [Konicki and Holman, 2000; Ribas and Kroon, 2007] is that the conditions suitable for bar growth (i.e., $\theta^{\text {off }}>30^{\circ}$ ) are less frequent at Duck (Table 1).

[39] A limitation of the model-data comparison of the previous paragraph is that the wave conditions have been varied in the model runs while keeping the profile constant, whereas in the field the profiles also differed in each event. The restricted amount of available measured profiles has also prevented us from validating the modeled sensitivity of bar characteristics on the underlying profile (Figure 10) against observations. A possible explanation for the disagreement between modeled and measured migration rates is that bar migration in the field is basically a finite-amplitude phenomenon, so the migration speed should be compared with the outcome of a non-linear model. For instance, the finite-amplitude up-current oriented bars obtained by Garnier et al. [2006] migrated at half the rate of what they did during their initial formation. Finally, note that tides were not included in the model, although Noordwijk beach is affected by semi-diurnal tides with a range of $1.6 \mathrm{~m}$ on average. Tides might have a significant effect on the formation of transverse finger bars since their growth time is of the same order of the timescale of tidal variability.

\subsection{Robustness and Limitations of the Model}

[40] Our model has been designed in such a way that it contains a large number of physical processes that are known to be important in the nearshore zone. Results show that the present model is able to capture conditions at our field site much better than an earlier model [Ribas et al., 2003], which was based on stricter assumptions. Setting up our model required parameterizations of complex processes and decisions had to be made to ignore certain interactions. It is thus important to discuss the robustness of the model with respect to changes in parameterizations and to processes that were excluded.

[41] We first emphasize that the key result of our study is that two conditions are necessary for growth of up-current oriented bars in the inner surf zone. These are that the depthaveraged sediment concentration decreases in the offshore direction and that the longshore current is deflected offshore over the bars. In our model, the first condition is met because of sediment resuspension induced by wave rollers. In principle, other phenomena could have the same effect, for example, stirring of sediment by low-frequency waves [cf. Aagaard and Hughes 2006]. The primary source for offshore deflection of currents over the bars turns out to be the torques resulting from depth varying frictional forces experienced by the wave-driven currents. In this respect, rollerinduced radiation stresses play an interesting, even double, role, but they are not essential.

[42] All in all, this suggests that our model is robust in the sense that changes in parameterizations (e.g. for bed shear stress, wave energy dissipation, sediment transport) and values of parameters (e.g. apparent roughness $k_{a}$, angle of roller interface $\beta_{\text {rol }}$, bedslope parameter $\gamma$ ) do not result in qualitative changes of the results, as long as the two conditions above are obeyed. We conduced many sensitivity experiments, which indeed confirm this statement.
[43] Our model uses depth-averaged equations of motion. This implies that three-dimensional phenonema, such as undertow and flow curvature, are not taken into account. It would certainly be interesting to study the formation of transverse bars with a three-dimensional model. Recent studies [cf. Kumar et al., 2011, and references therein] show that our knowledge of nearshore dynamics has sufficiently advanced to allow such studies in the near future.

[44] A final aspect that deserves discussion is that we neglected perturbations in the depth-integrated sediment concentration (equation (12)), also called sediment stirring. Experiments in which these perturbations were maintained resulted in output that was highly sensitive to numerical parameters (number of collocation points and their distribution over the computational domain). Our findings are in line with studies by Lane and Restrepo [2007] and Vis-Star et al. [2007], who studied the effect of perturbations in sediment stirring by waves on the initial formation of shoreface-connected sand ridges on the inner shelf and ended up with different conclusions. These results suggest that the presently available sediment transport formulations are not yet sufficiently accurate to correctly describe spatial variations in sediment stirring due to bedforms. This is not too surprising, as most formulations result from fitting data collected in laboratory settings to an empirical model.

\section{Conclusions}

[45] A new self-organization model based on linear stability analysis has been used to explain the main characteristics of surf zone transverse sandbars and reveal the physics behind their formation. The model describes the interaction between waves, rollers, depth-averaged currents and bed evolution so that feedback mechanisms can occur. The key aspect in order to explain the formation of the up-current oriented bars is the inclusion of the roller dynamics and the corresponding sediment resuspension due to roller-induced turbulence in the inner surf zone. The latter creates a depthaveraged sediment concentration decreasing seaward in that region, which is an essential ingredient for bar growth. This assumption is substantiated by previous studies that indicated that turbulence induced by breaking waves were the main mechanism for sediment resuspension in the inner surf zone, prevailing over bottom boundary layer processes.

[46] Model parameters are chosen to reproduce the beach conditions at Noordwijk beach, the Netherlands, and the results compare favorably with transverse bars observed there [Ribas and Kroon, 2007]. The wavelength (from 30 to $70 \mathrm{~m}$ ), up-current oriented shape and the e-folding growth time (about $12 \mathrm{~h}$ ) of the modeled bars are in good agreement with the observations. However, modeled migration speeds (tens of meters per day) are two times higher than those measured in the field. Both in the model and in the data, the wavelength of the bar patches scales with proxies for the inner surf zone width and their migration rate scales with the maximum of the longshore current in the inner surf zone.

[47] The model reveals that wave conditions needed for bar growth must be approximately constant during at least one day, with an offshore angle of wave incidence larger than $30^{\circ}$ and an offshore wave height larger than $0.25 \mathrm{~m}$. These modeled wave conditions for bar growth together with the low frequency of occurrence of large wave heights at 
Noordwijk provide a quantitative explanation of the wave conditions measured during the presence of bars by Ribas and Kroon [2007]: obliquely incident waves of intermediate heights. The annual mean conditions at Noordwijk are within this range, and this explains why transverse bar presence is so common there (compared with Duck beach, USA). Bathymetric profiles also affect bar characteristics but the corresponding model results can not be compared with observations due to the limited bathymetric surveys in the field. Thereby, performing bathymetric surveys during transverse bar presence is an essential future step in order to fully understand transverse bar formation.

[48] The present results show that a modified version of the bed-flow interaction, first studied in detail by Ribas et al. [2003], can be responsible for the development of observed up-current oriented bars. Positive feedback occurs if the depth-averaged sediment concentration decreases in the seaward direction because this enhances the convergence of sediment flux in the offshore-directed flows that occur over the growing bars. Such current perturbations are only induced in case of obliquely incident waves and it is essential the presence of a strong longshore current, which is deflected offshore over up-current oriented bars due to frictional torques. The roller radiation stresses play a double role. On the one hand, they damp the instability because the roller energy density is larger over the bars (more wave breaking in the shallows) and decrease toward the shoreline, and the opposite happens at the troughs. This produces cross-shore directed forces that oppose the current perturbations that are required for bar growth. At the same time, another roller-induced mechanism contributes to create the offshore-directed flows over the bars. The angle of wave incidence is larger at the shoreward end of the bars (stronger wave refraction in the shallows) and decrease toward the shoreline, and the opposite happens at the troughs. This produces forces in the alongshore direction that create alongshore directed currents near the shore (feeders), which converge and flow offshore over bar crests. If the angle of wave incidence in the inner surf zone is below a certain threshold value, the two mechanisms contributing to create an offshore deflection of the current over the bars are less efficient and the increased wave breaking over the bars reverses the current deflection so that transverse bars do not grow.

[49] Acknowledgments. The work of F. Ribas was partially supported by the Spanish government through the program 'Programa Juan de la Cierva'. We also acknowledge funding from the Spanish research projects 'Morfodinámica de playas: Predicciones en las grandes escalas espacio-temporales' (contract CTM2006-08875/MAR), 'Efectos de riadas y tormentas en los sedimentos y metales pesados asociados de un sistema litoral afectado por actividades industriales y urbanas' (contract CTM200606919), and 'Modelización y monitorización integradas en morfodinámica de playas naturales y regeneradas' (contract CTM2009-11892). The authors extend their sincere appreciation to the three reviewers, the associate editor and the editor, who provided comments that improved the quality of this paper.

\section{References}

Aagaard, T., and M. Hughes (2006), The influence of bore turbulence on sediment transport in the swash and inner surf zones, Mar. Geol., 228, 117-135.

Butt, T., P. Russel, J. Puleo, J. Miles, and G. Masselink (2004), The influence of bore turbulence on sediment transport in the swash and inner surf zones, Cont. Shelf Res., 24, 757-771.

Calvete, D., N. Dodd, A. Falqués, and S. M. van Leeuwen (2005), Morphological development of rip channel systems: Normal and near normal wave incidence, J. Geophys. Res., 110, C10006, doi:10.1029/ 2004JC002803.

Calvete, D., G. Coco, A. Falqués, and N. Dodd (2007), (Un)predictability in rip channel systems, Geophys. Res. Lett., 34, L05605, doi:10.1029/ 2006GL028162.

Falqués, A., A. Montoto, and V. Iranzo (1996), Bed-flow instability of the longshore current, Cont. Shelf Res., 16(15), 1927-1964.

Feddersen, F., R. T. Guza, S. Elgar, and T. H. C. Herbers (2000), Velocity moments in alongshore bottom stress parameterizations, J. Geophys. Res., 105(C4), 8673-8686.

Garnier, R., D. Calvete, A. Falqués, and M. Caballeria (2006), Generation and nonlinear evolution of shore-oblique/transverse sand bars, J. Fluid Mech., 567, 327-360.

Klein, M. D., and H. M. Schuttelaars (2005), Morphodynamic instabilities of planar beaches: sensitivity to parameter values and process formulations, J. Geophys. Res., 110, F04S18, doi:10.1029/2004JF000213.

Komar, P. D. (1998), Beach Processes and Sedimentation, 2nd ed., Prentice Hall, Englewood Cliffs, N. J.

Konicki, K. M., and R. A. Holman (2000), The statistics and kinematics of transverse bars on an open coast, Mar. Geol., 169, 69-101.

Kumar, N., G. Voulgaris, and J. Warner (2011), Implementation and modification of a three-dimensional radiation stress formulation for surf zone and rip-current applications, Coastal Eng., 58, 1097-1117.

Lane, E., and J. Restrepo (2007), Shoreface-connected ridges under the action of waves and currents, J. Fluid Mech., 582, 23-52.

Lippmann, T. C., A. H. Brookins, and E. B. Thornton (1996), Wave energy transformation on natural profiles, Coastal Eng., 27, 1-20.

Reniers, A. J. H. M., J. A. Roelvink, and E. B. Thornton (2004), Morphodynamic modeling of an embayed beach under wave group forcing, J. Geophys. Res., 109, C01030, doi:10.1029/2002JC001586.

Ribas, F., and A. Kroon (2007), Characteristics and dynamics of surfzone transverse finger bars, J. Geophys. Res., 112, F03028, doi:10.1029/ 2006JF000685

Ribas, F., A. Falqués, and A. Montoto (2003), Nearshore oblique sand bars, J. Geophys. Res., 108(C4), 3119, doi:10.1029/2001JC000985.

Ribas, F., H. de Swart, D. Calvete, and A. Falqués (2011), Modeling waves, currents and sandbars on natural beaches: The effect of surface rollers, J. Marine Syst., 88, 90-101, doi:10.1016/j.jmarsys.2011.02.016.

Roelvink, J. A., and M. J. F. Stive (1989), Bar-generating cross-shore flow mechanisms on a beach, J. Geophys. Res., 94(C4), 4785-4800.

Ruessink, B. G., J. R. Miles, F. Feddersen, R. T. Guza, and S. Elgar (2001), Modeling the alongshore current on barred beaches, J. Geophys. Res., 106(C10), 22,451-22,463.

Soulsby, R. L. (1997), Dynamics of Marine Sands, Thomas Telford, London, U. K.

Svendsen, I. (1984), Mass flux and undertow in the surf zone, Coastal Eng., $8,347-365$

Thornton, B., and R. T. Guza (1983), Transformation of wave height distribution, J. Geophys. Res., 88(10), 5925-5938.

Thornton, B., and R. T. Guza (1986), Surf zone longshore currents and random waves: Field data and models, J. Phys. Oceanogr., 16, 1165-1178.

Trowbridge, J. H. (1995), A mechanism for the formation and maintenance of shore-oblique sand ridges on storm-dominated shelves, J. Geophys. Res., 100(C8), 16,071-16,086.

van Enckevort, I. M. J., B. G. Ruessink, G. Coco, K. Suzuki, I. L. Turner, N. G. Plant, and R. A. Holman (2004), Observations of nearshore crescentic sandbars, J. Geophys. Res., 109, C06028, doi:10.1029/ 2003JC002214.

van Leeuwen, S. M., N. Dodd, D. Calvete, and A. Falqués (2006), Physics of nearshore bed pattern formation under regular or random waves, J. Geophys. Res., 111, F01023, doi:10.1029/2005JF000360.

Vis-Star, N., H. de Swart, and D. Calvete (2007), Effect of wave-topography interactions on the formation of sand ridges on the shelf, J. Geophys. Res., 112, C06012, doi:10.1029/2006JC003844.

Vis-Star, N., H. de Swart, and D. Calvete (2008), Patch behaviour and predictability properties of modelled finite-amplitude sand ridges on the inner shelf, Nonlin. Processes Geophys., 15, 943-955.

Voulgaris, G., and M. B. Collins (2000), Sediment resuspension on beaches: response to breaking waves, Mar. Geol., 167, 167-187.

Wright, L. D., and A. D. Short (1984), Morphodynamic variability of surf zones and beaches: A synthesis, Mar. Geol., 56, 93-118.

Zimmerman, J. T. F. (1981), Dynamics, diffusion and geomorphological significance of tidal residual eddies, Nature, 290, 549-555.

D. Calvete, A. Falqués, and F. Ribas, Departament de Física Aplicada, Universitat Politècnica de Catalunya, Campus Nord, Jordi Girona 1-3, E-08034 Barcelona, Spain. (francesca.ribas@upc.edu)

H. E. de Swart, Institute for Marine and Atmospheric Research Utrecht, Utrecht University, Princetonplein 5, NL-3584 CC Utrecht, Netherlands. 